\title{
Influence of Different Statuses of Honey Bee Queens, Apis mellifera L. on the Ultrastructure of the Flagella on (3-Day Old) Workers
}

\author{
Azza A. Awad ${ }^{1, *}$, Adham M. Moustafa ${ }^{2}$, Mohamed F. Abdel- Rahman² and Rania Q. Sayed ${ }^{2}$ \\ ${ }^{I}$ Zoology Department, Faculty of Science, Assiut University; ${ }^{2}$ Plant Protection Res. Institute, Agricultural Research \\ Center, Giza, Egypt
}

\begin{abstract}
The effect of different statuses of honeybee queens, Apis mellifera (one-year-old mated queen; supersedure queen; three-day old virgin queen; queen cell; without queen; and without queen/without bee bread) on many aspects of 3day old workers were studied with scanning electron microscopy (SEM). This study was carried out to describe the (types, distribution, numbers and measurements) of the different sensilla on the workers' flagellum. Electrone microscopic study of workers under different queen statuses showed the morphological structure of the antennae (Geniculate antennae). Each antenna composed of scape, a pedicel, and a flagellum of 10 segments. SEM revealed the presence of seven types and eleven subtypes of sensilla on the dorsal side of the different flagellomeres; they were Sensilla Ampullacea $\{$ Am $\}$, Sensilla Basiconica $\{\mathrm{Ba}\}$, Sensilla Campaniformia $\{\mathrm{Cf}\}$, Sensilla Chaetica I, II $\{\mathrm{Ch}\}$, Sensilla Coelloconica I, II $\{\mathrm{Co}\}$, Sensilla Placodea I, II, III $\{P L\}$, Sensilla Trichodea I, II, III, IV $\{\mathrm{Tr}\}$. This study revealed that, there is no significant difference in the numbers of sensilla (Am), (Ba), (Co I) of the workers under all the queen statuses. The measurements of sensilla Chaetica differ significantly according to the different queen statuses. The numbers and distributions of (Co II) were exhibited at extremely low percentages in the different statuses. There is significant difference in the percentages of each (PL I, II, III) between the different queen statuses. Also, the (Tr I, II, III, IV) are found in all statuses, There is significant difference in the percentages of each sensilla types between the different queen statuses.
\end{abstract}

Keywords: Apis mellifera L., flagella, honey bee, morphology, queen statuses, scanning electron microscopy (SEM), sensory structure.

\section{INTRODUCTION}

The honeybee queen is a very important member in her colony; its primary function is reproduction. The queen produces both fertilized and unfertilized eggs; it is capable to produce more than 1500 eggs a day during peak production. The second major function of the queen is producing pheromones. One major pheromone termed as queen substance is produced by her mandibular glands, but many other phermones are also important. The qualities of the colony depend largely on the egg-laying and chemical production capabilities of the queen [1].

Honeybees from different castes have different functions in their colony and exhibit different external and internal morphology. This is especially true for the antennae and for the antennal sensilla. For example, honeybee queens use their antennal sensilla to perceive colony odors, while workers use their antennal sensilla to detect odors such as queen pheromone, brood pheromone and floral perfumes, workers can distinguish the odors of plants in bloom. For drones, the most important use for their olfactory sensilla is to detect queen pheromone [2].

Workers may change between different tasks depending on their physiological maturation or age $[3,4]$.

*Address correspondence to this author at the Zoology Department, Faculty of Science, Assiut University, Egypt; Tel: 0882412071;

Fax: 0882342708; E-mail: awadazza@yahoo.com
In the Apis mellifera, for instance, young individuals perform in-hive tasks (e.g., nursing, comb construction); as they age, bees move to tasks at the hive entrance (e.g., guarding) and eventually switch to activities outside of the hive (e.g., foraging) [5]. On the other hand, worker specialization may be associated with morphological and anatomical variation between individuals (physical castes) [6].

Queen-worker interactions in the honeybee colony are mostly pheromone-mediated (Pheromones are chemicals that elicit behavioral and physiological responses in conspecific individuals or groups). The queen pheromones control workers morphology $[7,8]$.

SEM studies of worker, A. mellifera, have been carried out earlier by $[9,10]$ and also on many other Hymenopterans $[11,12]$. The number and distributions of the different types of sensilla on antennae A. florea F. were examined by [13, 14]. He found that, the distribution of various types of sensilla along the antenna is similar to that found in other Apis species. The highest density of sensilla was found on the distal antennal segment.

During the first days of adult life; more and more bees join the retinue of the queen $[15,16]$, i. e. their behavior changes. Improvements in olfactory learning performance during the first days of adult life correlate in time with the increased sensitivity of workers' antennal receptors to queen pheromone [17] and other olfactory stimuli [18, 19]. 
Bees showed a poor learning performance to odours only during the first 2 days of age; later workers achieved a higher level of acquisition, i.e. on the $3^{\text {rd }}-4^{\text {th }}$ day it became equal to that of older bees [20-23]. During the first week of workers' life, the sensilla Placodea [24] and the neuropil of the olfactory centre [25] undergo changes.

The objective of the current study is to determine the effect of different statuses of honeybee queens, Apis mellifera (one-year-old mated queen; supersedure queen; three-dayold virgin queen; queen cell; without queen; and without queen/without bee bread) on many aspects of three-day old workers including morphology and behavior as following:

- Identifying the different types of the sensilla focusing on their distribution, abundances and measurements on the different flagellomeres (I, III, V, VII, and IX) of the workers.

\section{MATERIALS AND METHODS}

The experiments were carried out in Assiut, Insect Research Laboratory, Plant Protection Research Institute during the active season of 2012 .

\subsection{Preparation of Bee Cages and Bioassay Protocol}

The first hybrid of Carniolan honeybee, Apis mellifera carnica Pollmann workers were used in the present study. Sealed brood combs, containing hatching brood, were taken from queen right colony, then incubated at $32^{\circ} \mathrm{C} \pm 1$ and $60 \%$ $\mathrm{RH}$. and the brood were observed until adults emergence. Experimental wooden cages of $(15 \times 15 \times 5 \mathrm{~cm}$. $)$ dimensions with a glass side and other was covered with black muslin (Fig. 1). Every cage was provided with a vial of tap water and other vial of sugar solution 1:1 (w: v); bee bread and a pieces of wax foundation (wax sheet embossed with the shape of cell bases), were attached to the cage side, each wax foundation with dimensions $9.5 \times 5 \mathrm{~cm}$. $\left(\right.$ area $\left.=47.5 \mathrm{~cm}^{2}\right)$ containing 180 base of a worker cells. Newly emerged workers aged $0-12$ hours were confined in the experimental cages (200 workers / cage as a replicate).

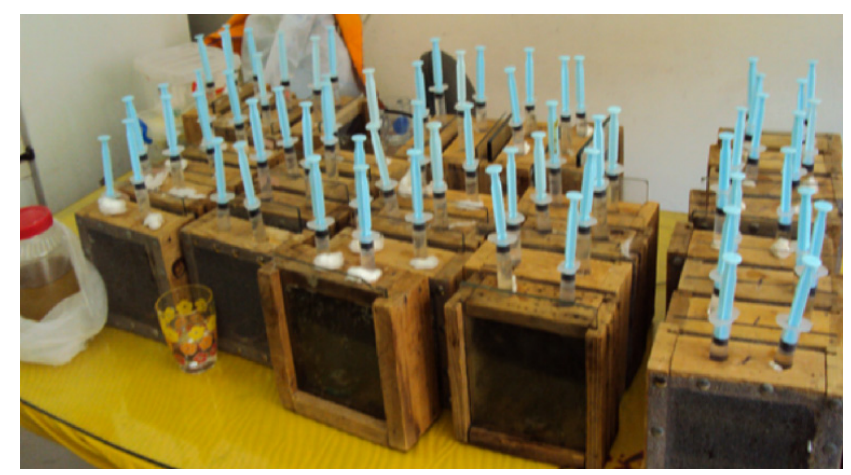

Fig. (1). Experimental wooden cages of $(15 \times 15 \times 5 \mathrm{~cm})$ dimensions with a glass side and other was covered with black muslin. Every cage was provided with a vial of tap water and other vial of sugar solution 1:1 (w: v); bee bread and a pieces of wax foundation were attached to the cage side.

The cages were continuously supplied with water, sucrose solution and bee bread, they were divided into six groups dependent on status of the introduced queens as follows:
- Group « 1 〉, cages contained one-year-old mated and egglaying queens: (M).

- Group ‘ 2 〉, cages contained mated and egg-laying queens that replaced by Honey bees (supersedure queens): (S).

- Group $<3$ >, cages contained three-day-old virgin queens: (V).

- Group \& 4 〉, cages contained queen cells at beginning of sealed: $(\mathrm{Q})$.

- Group 〈 5 〉, cages without queens (queenless) as a control: $(\mathrm{W})$.

- Group $\langle 6\rangle$, cages without queens / without bee bread: (W/W).

Four replicates were used for each group. The cages were held in a dark incubator at $32{ }^{\circ} \mathrm{C} \pm 1$ and $60 \% \mathrm{RH}$.

\section{A. Determination of Morphological \& Ultrastructural Studies of the Workers Antennae}

To study the effect of the different queen statuses on the types, distribution, numbers and measurements of the different sensilla on the workers' flagellum; about 5-7 workers were removed from each cage (Fig. 2). This procedure was repeated six times. The head of each worker was removed to examine the antennae and studied it morphologically and ultrastructurally by scanning electron microscope (SEM) (JEOL 5400LV. in SEM Unit, Assuit University).

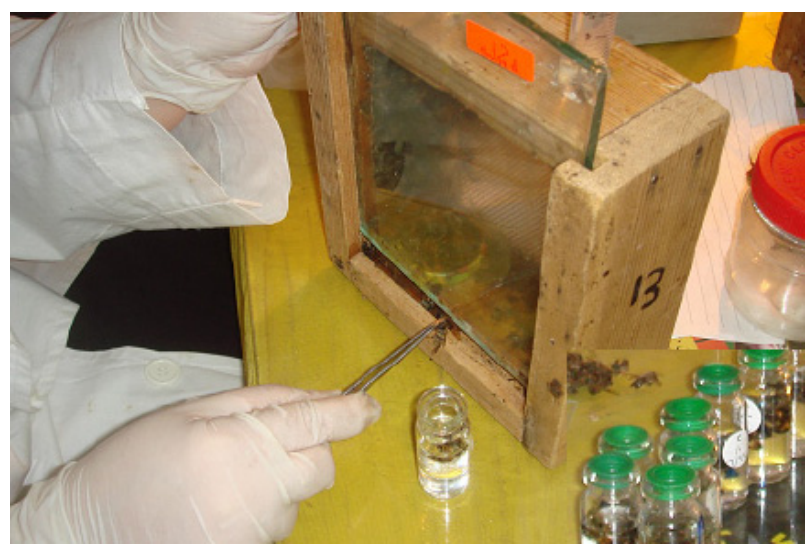

Fig. (2). Workers were removed from each cage every three days to examine the antennae and studied it morphologically and ultrastructurally by (SEM).

In this paper; in each worker, one antenna (left or right) was examined. The workers' flagellomeres were numbered I, II, III, IV ,.......IX, X, beginning distally. The singles flagellomeres (I, III, V, VII, and IX) were examined from the dorsal side per unit area $(122 \mu \mathrm{m} \times 82 \mu \mathrm{m})$ by SEM.

Scanning electron microscopy (SEM) was done as described by Azza Awad [26] and the five following steps have been followed:

\section{A.1. Fixation}

Khal's Solution Fixation (Using Freeze- Drying)

In this study, Khal's solution has been used as a fixative and it was prepared as follows: $30 \mathrm{ml}(95 \% \mathrm{EtOH})+12 \mathrm{ml}$ 
formaldehyde $+4 \mathrm{ml}$ glacial acetic acid $+60 \mathrm{ml}$ distilled water. Specimens can be ready for fixation directly after mixing these components. Fixation by Khal's solution should last at least 7-8 days.

\section{A.2. Dehydration}

After fixation, the fixative is washed by three washes in the same buffer vehicle as used for the fixative. Ethanol is the most widely used dehydration agent. After secondary fixation, specimens should be left in a series of ascending alcohols (30\%, 50\%, 70\%, 90\% (two washes), 100\% (3 or 4 washes) each for 2 hours), in order to eliminate the small amount of water remaining in the tissue. Then drain off excess alcohol and put in amylacetat for 1-2 days.

\section{A.3. Drying}

The method chosen for drying is dependent on the type of material to be examined. Insects because of their hard outer skeleton may be air-dried in room temberatute (35-37 ${ }^{\circ} \mathrm{C}$ ), for nearly one-two hours at maximum.

\section{A.4. Final Mounting}

After the specimen has been dried, it should be mounted on SEM holder called a specimen stub. The surface of the stub should be as smooth and free of structure as possible to prevent confusing backgrounds with sticky-taps (adhesive).

Specimen may then be carefully placed onto the adhesive, clean air has been used to press it into the adhesive.The specimen holder have been labeled from the underside with a permanent marker pen, and stored until it being ready for sputter coating.

\section{A.5. Sputter Coating}

The specimen then coated with gold film with $150 \mathrm{~A}^{\mathrm{o}}$ thickness using JEOL (JFC-1100E) sputtering device for 2-3 minutes. The specimen is now ready for examination by the scanning electron microscope, SEM (JEOL 5400LV. in SEM Unit, Assuit University) in the low vacuum mode at voltage $15 \mathrm{KV}$, at magnifications ranged from (X 50 to 1000) for surveys, and (X 1000 to 15000) for identifications of sensilla types.

Identification of sensilla types was carried out according to [27-30]. The sensilla were counted and measured according to the magnification force and depending on the morphological shape of the sensilla. We classified the different types of sensilla into seven types and eleven subtypes; sensilla Ampullacea $\{A m\}$, sensilla Basiconica $\{B a\}$, sensilla Campaniformia $\{\mathrm{Cf}\}$, sensilla Chaetica I, II $\{\mathrm{Ch}\}$, sensilla Coelloconica I, II $\{\mathrm{Co}\}$, sensilla Placodea I, II, III $\{\mathrm{PL}\}$, and sensilla Trichodea I, II, III, IV $\{\mathrm{Tr}\}$ (Micrograph 1). Hence the measurement of Basiconica, Chaetica, and Tricodea indicated by length in $\mu \mathrm{m}$ and the measurement of Ampullacea, Campaniformia, and Coeloconica indicated by diameter in $\mu \mathrm{m}$, but the measurement of Placodea calculated by area in $\mu \mathrm{m}^{2}$ and this according to the formula used by [31]:

$$
\text { Surface area }=\Pi \times \frac{a \times b}{2}
$$

Where:

$a=$ maximum length in $\mu \mathrm{m}, b=$ maximum width in $\mu \mathrm{m}$, and $\Pi=3.14$
Differences in sensillum numbers were analysed using analysis of variance (ANOVA) followed by least significant test (LSD) at $\mathrm{P}<0.05$. All statistical calculations were performed in SPSS.

\section{RESULTS}

\subsection{Morphological Characters of the Workers}

\section{A. Determination of Morphological \& Ultrastructural Studies of the Workers Antennae}

Electrone microscopic study of honeybee workers Apis mellifera at headed by different queen statuses (one-year old mated queen; supersedure queen; three-day-old virgin queen; queen cell; without queen; and without queen/without bee bread), showed the morphological structure of the antennae (Geniculate antennae). Each antenna consists of scape, pedicel, and flagellum of 10 segments, (Micrograph 2).

SEM revealed the presence of seven types and eleven subtypes of sensilla on the dorsal side of the different flagellomeres of the honeybee workers' antennae, (Micrograph 1); they were Sensilla Ampullacea $\{\mathrm{Am}\}$, Sensilla Basiconica $\{\mathrm{Ba}\}$, Sensilla Campaniformia $\{\mathrm{Cf}\}$, Sensilla Chaetica I, II $\{\mathrm{Ch}\}$, Sensilla Coelloconica I, II $\{\mathrm{Co}\}$, Sensilla Placodea I, II, III $\{P L\}$, Sensilla Trichodea I, II, III, IV $\{\mathrm{Tr}\}$.

Where, the classification of sensilla types in honeybee is done on the basis of cuticular morphology supported by ultrastructural and electrophysiological; also on the functional differences among these sensilla.

1) Sensilla Ampullacea $\{\mathrm{Am}\}$ appearing as deep holes or cavities in the antennal surface with small diameters, only about $1 \mu \mathrm{m}$, within which there is an inner process.

2) Sensilla Basiconica $\{\mathrm{Ba}\}$ are straight digitiform, Peg, cone, or finger-like structure sensilla with arounded porous tip; inserted into slightly depressed round socket.

3) Sensilla Campaniformia $\{\mathrm{Cf}\}$ or coelocapitular sensilla, are dome-like or bell-shaped cuticular areas, they are elevated above or depressed below the general cuticular surface, they comprise a cuticular, shallow depression having a central opening and a mushroom-shaped protrusion from the opening, the head of the protrusion is irregular in shape and is not perforated, they are found on at least 9 of 10 flagellomeres of honeybee workers.

4) Sensilla Chaetica $\{\mathrm{Ch}\}$ are stout bristles, or spine-like with their base inserted into a socket and very sharpened and tapering tips. They house an apical pore and they are similar to the sensilla Trichodea, but have thicker cuticular shafts and are not freely moveable. They could be divided into long (Chaetica I) and short (Chaetica II).

5) Sensilla Coelloconica $\{\mathrm{Co}\}$ : They could be divided into (Coelloconica I) that with very slightly protrusive peg and (Coelloconica II) with longest peg.

6) Sensilla Placodea $\{\mathrm{PL}\}$, or pore plate organs, they are oval to nearly circular disc with numerous pores, where they are surrounded by a multi-porous membranous ring, so they are distinguished as plate organs. They are located above, or below the cuticular surface. They are divided into open Placodea surrounded by an outer membranous ring, positioned below the cuticular surface 

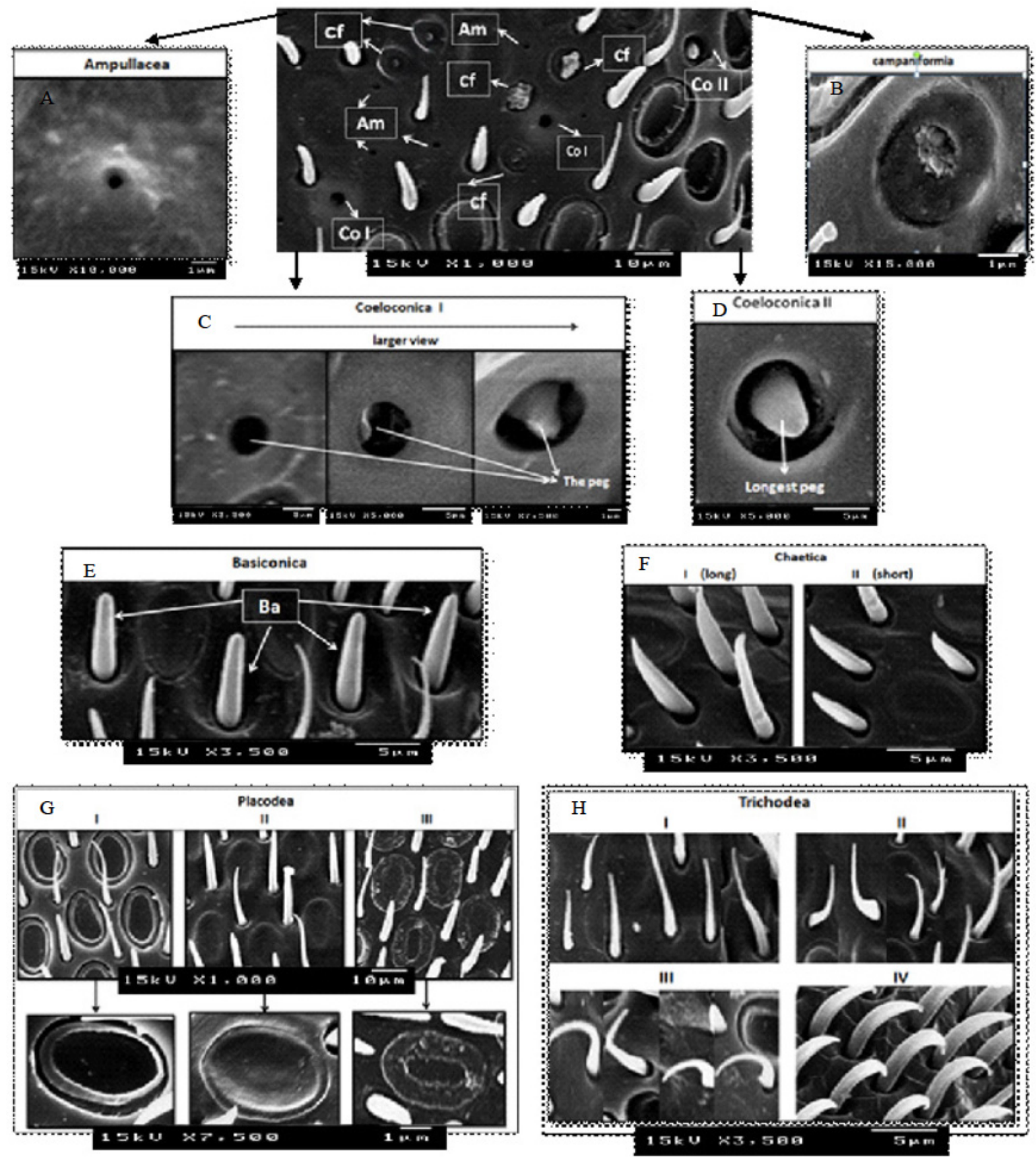

Micrograph (1). The different sensilla types on the flagellum of honeybee workers Apis mellifera, [A]: sensilla Ampullacea (Am); [B]: sensilla Campaniformia (Cf); [C \& D]: sensilla Coelloconica (Co I, II); [E]: sensilla Basiconica (Ba); [F]: sensilla Chaetica (Ch I, II); [G]: sensilla Placodea (PL I, II, III); and [H]: sensilla Trichodea (Tr I, II, III, IV).

(type I); closed Placodea covered externally by an oval or elliptical plate surrounded by a narrow outer membranous ring, positioned at the cuticular surface (type II); and closed Placodea covered externally by an oval or elliptical plate surrounded by wider outer membranous ring, positioned above the cuticular surface (type III), they are located on the last eighth distal flagellomeres of the honeybee workers.

7) Sensilla Trichodea $\{\operatorname{Tr}\}$ are hair-like structure (seta), which vary greatly in length and are freely moveable. They could be divided into straight thin Trichodea (type
I), slightly curved thin Trichodea (type II), strongly curved and arched thin Trichodea (type III) and thick Trichodea that are slightly curved (type IV).

\section{A.1. Workers at three-day old under different queen statuses.}

The numbers and measurements of antennal sensilla per unit area $(122 \mu \mathrm{m} \times 82 \mu \mathrm{m})$ on the dorsal side of different flagellomeres of Apis mellifera workers at three-day-old under different queen statuses are presented in (Table 1A \& B), and (Fig. 3; \& Micrograph 3A-F). 


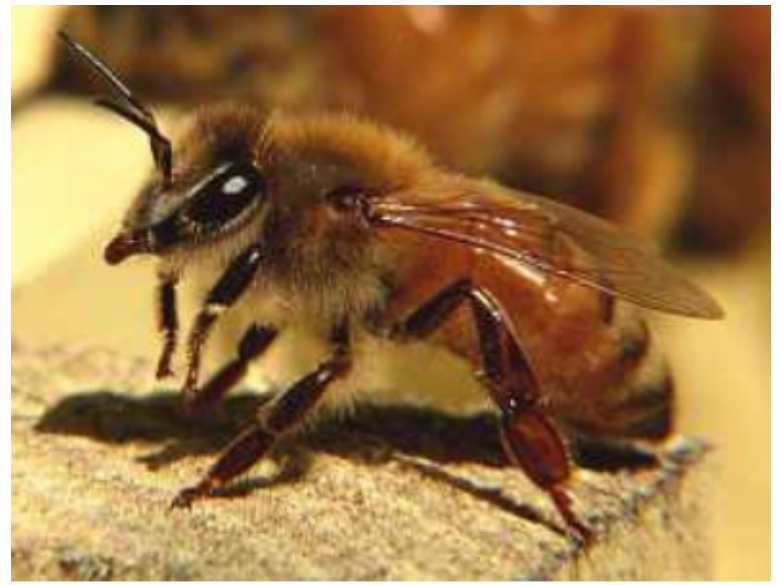

Honey bee worker, Apis mellifera

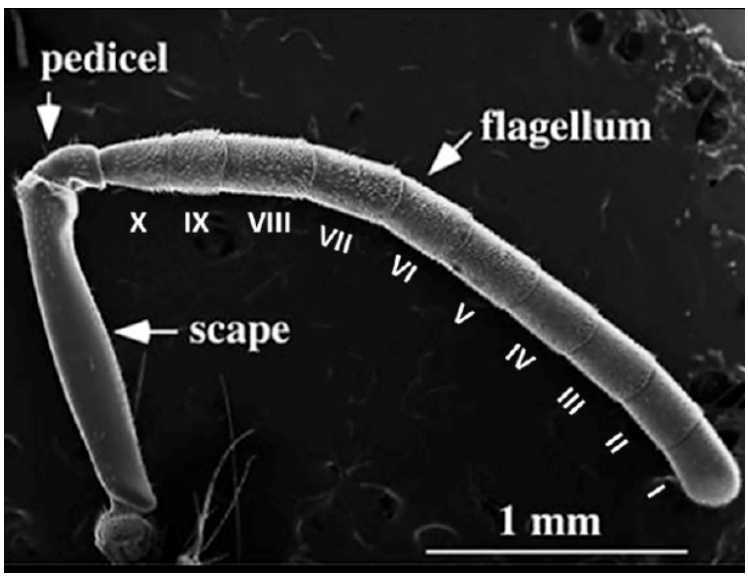

Micrograph (2). Scanning Electron microscope (SEM) showing the morphological structure of the antenna of the honeybee workers Apis mellifera (Geniculate antenna). Each antenna consists of scape, pedicel, and a long slender flagellum, which is composed of 10 segments in the workers.

Ampullacea sensilla: on the flagellum segment of the worker on virgin queen and without queen and without bee bread statuses have no significant differences $(P>0.2)$ comparing with those in the case of one year old mated queen. While, these types of sensilla of the worker in supersedure, queen cell at the beginning of sealed, and without queen comparing by those in the control statuse which is one year old mated queen is highly significant difference $(P<0.01)$.

Basiconica sensilla: comparing with sensilla in the case of one year old mated queen to those on virgin queen and supersedure queen statuses there is no significant differences $(P>0.9)$. While, these types of sensilla of the worker in queen cell at the beginning of sealed, without queen, and without queen and without bee bread comparing by those in the control statuse is highly significant difference $(P<0.01)$.

Companiformia and trichodia sensilla (TrII): there is no significant differences $(P>0.3)$ on sensilla of the worker on virgin queen, without queen and without bee bread, and supersedure queen statuses if comparied with those in the case of one year old mated queen. While, these types of sensilla in queen cell at the beginning of sealed, and without queen comparing by those in the control statuse is highly significant difference $(P<0.01)$.
Cheatica sensilla (ChI): sensilla on the flagellum segment of the worker on without queen and without bee bread statuses showing no significant differences $(P>0.9)$ comparing with those in the case of one year old mated queen. On the other hand, these types of sensilla of the worker in supersedure, queen cell at the beginning of sealed, and without queen comparing by those in the control statuse is significant difference $(P<0.05)$.

Cheatica sensilla (ChII) \& Coelloconica I: on the worker of supersedure queen statuses, there is no significant differences $(P>0.8)$ comparing with those in the case of control statuse. While, these types of sensilla of the worker in queen cell at the beginning of sealed, without queen , and without queen and without bee bread comparing by those in the control statuse represent highly significant difference $(P$ $<0.01)$.

Placodea sensilla I: sensilla on the worker in supersedure, queen cell at beginning of sealed, virgin queen, and without queen and without bee bread comparing by those in the control statuse has highly significant difference $(P<$ $0.01)$. Contrary to them of the worker on without queen statuses have no significant differences $(P>0.1)$.

Placodea sensilla II \& Trichodia III sensilla: on the flagellum segment of the worker in all queen statuses comparing by those in the control statuse which is one year old mated queen is highly significant $(P<0.01)$.

Trichodia I sensilla: on the worker of virgin queen, supersedure, queen cell at the beginning of sealed, and without queen and without bee bread statuses, there are no significant differences $(P>0.4)$ in compare with those in the case of one year old mated queen. While, these types of sensilla of the worker in without queen comparing by those in the control statuse $(P<0.01)$.

Trichodia IV sensilla: there are a significant difference $(P<0.05)$ on sensilla of the worker in queen cell at the beginning of sealed, and without queen comparing by those in the control statuse. Where, on the worker of virgin queen, supersedure, and without queen and without bee bread statuses show no significant differences $(P>0.4)$.

We quantified the different types of sensilla to calculate the average percentage of each sensilla type, on the five single flagellomeres,

According to, (Table 1A \& B;), and (Fig. 3; \& Micrograph 3A-F), we could concloude the following results:

a- Sensilla Ampullacea (Am) didn't appear on the different flagellomeres in the queen cell status, but a few numbers of these sensilla were distributed in the other statuses, the measurements of $(\mathrm{Am})$ were about $0.667 \mu \mathrm{m}$ in diameter in the statuses where they appeared.

b- Sensilla Basiconica (Ba) coexisted on the third, fifth and seventh flagellomeres in the one-year-old mated queen, supersedure queen, and three-days-old virgin queen statuses, with their number increasing distally; they presented only on the fifth flagellomere (queen cell status); the fifth and seventh (without queen status); and the first and seventh (without queen/without bee bread status). The (Ba) were absent on the ninth flagellomere in all statuses. The measurement of $(\mathrm{Ba})$ ranged from about 6 to $11.67 \mu \mathrm{m}$ in length in all statuses. 
Table 1A. Mean \pm standard error of antennal sensilla per unit area $(122 \mu \mathrm{m} \times 82 \mu \mathrm{m})$ on the dorsal side of different flagellomeres of Apis mellifera workers at three-day-old under different queen statuses.

\begin{tabular}{|c|c|c|c|c|c|c|}
\hline $\begin{array}{c}\text { Queen Statues Types } \\
\text { of Sensilla }\end{array}$ & $\begin{array}{c}\text { One-Year-Old } \\
\text { Mated Queen }\end{array}$ & $\begin{array}{c}\text { Supersedure } \\
\text { Queen }\end{array}$ & $\begin{array}{c}\text { Three-Days-Old } \\
\text { Virgin Queen }\end{array}$ & $\begin{array}{c}\text { Queen Cell at } \\
\text { Beginning of } \\
\text { Sealed }\end{array}$ & $\begin{array}{c}\text { Without Queen } \\
\text { Without Queen } \\
\text { andthout Bee } \\
\text { Bread }\end{array}$ \\
\hline \hline Ampullacea (Am) & $10 \pm 1.73^{\mathrm{a}}$ & $11 \pm 0.58^{\mathrm{a}}$ & $2 \pm 0.58^{\mathrm{b}}$ & $0 \pm 0^{\mathrm{b}}$ & $2 \pm 0.58^{\mathrm{b}}$ & $13 \pm 1.73^{\mathrm{a}}$ \\
\hline Basiconica (Ba) & $21 \pm 3.46^{\mathrm{a}}$ & $19 \pm 1.15^{\mathrm{a}}$ & $20 \pm 3.46^{\mathrm{a}}$ & $5 \pm 1.15^{\mathrm{b}}$ & $8 \pm 0.58^{\mathrm{b}}$ & $4 \pm 0.58^{\mathrm{b}}$ \\
\hline Campaniformia (Cf) & $10 \pm 1.73^{\mathrm{ab}}$ & $8 \pm 0.58^{\mathrm{b}}$ & $12 \pm 0^{\mathrm{a}}$ & $0 \pm 0^{\mathrm{c}}$ & $9 \pm 0^{\mathrm{c}}$ & $14 \pm 0.58^{\mathrm{d}}$ \\
\hline Chaetica (Ch) I & $140 \pm 3.46^{\mathrm{a}}$ & $39 \pm 0^{\mathrm{c}}$ & $133 \pm 0.58^{\mathrm{a}}$ & $58 \pm 1.73^{\mathrm{b}}$ & $139 \pm 0.58^{\mathrm{a}}$ \\
\hline Chaetica (Ch) II & $98 \pm 2.89^{\mathrm{d}}$ & $232 \pm 4.04^{\mathrm{b}}$ & $96 \pm 0.58^{\mathrm{d}}$ & $216.67 \pm 2.6^{\mathrm{c}}$ & $296 \pm 0.58^{\mathrm{a}}$ & $58 \pm 0.58^{\mathrm{e}}$ \\
\hline Coeloconica I & $12 \pm 1.73^{\mathrm{a}}$ & $7 \pm 1.15^{\mathrm{ab}}$ & $10.33 \pm 1.76^{\mathrm{a}}$ & $2 \pm 0^{\mathrm{bc}}$ & $0 \pm 0^{\mathrm{c}}$ & $4 \pm 1.73^{\mathrm{bc}}$ \\
\hline Placodea I & $18 \pm 0.58^{\mathrm{d}}$ & $137 \pm 1.15^{\mathrm{b}}$ & $107 \pm 1.73^{\mathrm{c}}$ & $161 \pm 1.73^{\mathrm{a}}$ & $22 \pm 0^{\mathrm{d}}$ & $164 \pm 1.15^{\mathrm{e}}$ \\
\hline Placodea II & $117 \pm 5.2^{\mathrm{b}}$ & $45 \pm 1.73^{\mathrm{d}}$ & $78 \pm 3.46^{\mathrm{c}}$ & $70 \pm 0.58^{\mathrm{c}}$ & $257 \pm 0^{\mathrm{a}}$ & $55 \pm 0.58^{\mathrm{d}}$ \\
\hline Trichodea I & $30 \pm 2.31^{\mathrm{a}}$ & $27 \pm 5.2^{\mathrm{a}}$ & $36 \pm 2.89^{\mathrm{a}}$ & $24 \pm 1.73^{\mathrm{a}}$ & $6 \pm 1.15^{\mathrm{b}}$ & $32.33 \pm 1.2^{\mathrm{a}}$ \\
\hline Trichodea II & $44 \pm 1.15^{\mathrm{b}}$ & $45 \pm 1.73^{\mathrm{b}}$ & $41 \pm 1.15^{\mathrm{b}}$ & $56 \pm 1.15^{\mathrm{a}}$ & $33 \pm 0.58^{\mathrm{c}}$ & $41 \pm 1.15^{\mathrm{b}}$ \\
\hline Trichodea III & $12 \pm 2.31^{\mathrm{b}}$ & $21 \pm 1.73^{\mathrm{a}}$ & $2 \pm 0^{\mathrm{c}}$ & $3 \pm 1.15^{\mathrm{c}}$ & $20 \pm 1.73^{\mathrm{a}}$ & $5 \pm 0.58^{\mathrm{c}}$ \\
\hline Trichodea IV & $51 \pm 0.58^{\mathrm{b}}$ & $82 \pm 1.15^{\mathrm{ab}}$ & $84 \pm 0.58^{\mathrm{ab}}$ & $149.33 \pm 35.86^{\mathrm{a}}$ & $117 \pm 1.73^{\mathrm{ab}}$ & $66 \pm 0.58^{\mathrm{b}}$ \\
\hline
\end{tabular}

The values for each type of sensilla within the queen statusese followed by the same letter are not significantly different $(P<0.05)$.

Table 1B. Measurements of antennal sensilla per unit area $(122 \mu \mathrm{m} \times 82 \mu \mathrm{m})$ on the dorsal side of the different flagellomeres of Apis mellifera worker at three-day-old under different queen statuses.

\begin{tabular}{|c|c|c|c|c|c|c|c|c|c|c|c|c|c|c|c|c|c|c|c|c|c|c|}
\hline \multirow{4}{*}{ 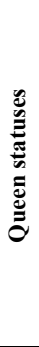 } & \multirow{4}{*}{ 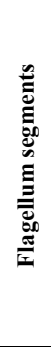 } & \multicolumn{21}{|c|}{ Measurements of sensilla organs } \\
\hline & & \multirow{3}{*}{ 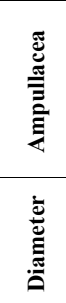 } & \multirow{3}{*}{ 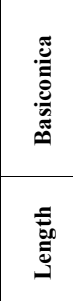 } & \multirow{3}{*}{ 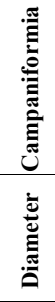 } & \multicolumn{2}{|c|}{ Chaetica } & \multicolumn{3}{|c|}{ Coeloconica } & \multicolumn{9}{|c|}{ Placodea } & \multicolumn{4}{|c|}{ Tricodea } \\
\hline & & & & & \multirow{2}{*}{ 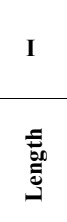 } & \multirow{2}{*}{$\begin{array}{l}\text { II } \\
\text { E્ } \\
\text { Е }\end{array}$} & \multirow{2}{*}{ 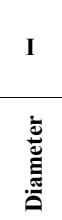 } & \multicolumn{2}{|c|}{ II } & \multicolumn{3}{|c|}{ I } & \multicolumn{3}{|c|}{ II } & \multicolumn{3}{|c|}{ III } & \multirow{2}{*}{ 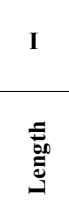 } & \multirow{2}{*}{ 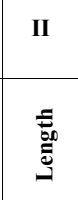 } & \multirow{2}{*}{ 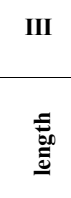 } & \multirow{2}{*}{ 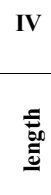 } \\
\hline & & & & & & & & 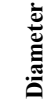 & 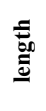 & 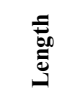 & $\frac{E}{3}$ & $\underset{d}{\stackrel{J}{Z}}$ & 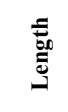 & 吾 & $\stackrel{\mathbb{Z}}{Z}$ & 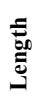 & $\frac{5}{E}$ & 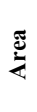 & & & & \\
\hline \multirow{5}{*}{ 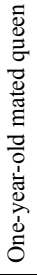 } & I & 0 & 0 & 0 & 12.67 & 9 & 0 & 0 & 0 & 11 & 8.33 & 143.91 & 10.13 & 7.73 & 122.94 & 0 & 0 & 0 & 12 & 12.8 & 14.4 & 0 \\
\hline & III & 0.667 & 10.67 & 4.33 & 13.47 & 8.4 & 2.13 & 0 & 0 & 12.67 & 7.73 & 153.73 & 12.4 & 8.27 & 161.00 & 0 & 0 & 0 & 12 & 14.4 & 13.78 & 0 \\
\hline & V & 0.667 & 8.53 & 4.33 & 13.33 & 8.8 & 2.33 & 0 & 0 & 11 & 8 & 138.16 & 12.53 & 8.27 & 162.69 & 0 & 0 & 0 & 11.13 & 12.4 & 0 & 0 \\
\hline & VII & 0.667 & 9.73 & 4.33 & 14.8 & 9.6 & 2 & 0 & 0 & 12.67 & 7.55 & 150.18 & 12.93 & 9.33 & 189.39 & 0 & 0 & 0 & 12.8 & 12.27 & 13.33 & 0 \\
\hline & IX & 0 & 0 & 0 & 16.67 & 0 & 0 & 0 & 0 & 0 & 0 & 0 & 0 & 0 & 0 & 0 & 0 & 0 & 14.33 & 0 & 0 & 19.2 \\
\hline \multirow{5}{*}{ 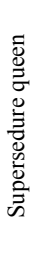 } & I & 0.667 & 0 & 0 & 13.07 & 9.2 & 0 & 2.67 & 2 & 11.47 & 6.8 & 122.45 & 10 & 5.67 & 89.02 & 0 & 0 & 0 & 11.33 & 10.67 & 0 & 0 \\
\hline & III & 0 & 11.33 & 4.53 & 16 & 8 & 2.4 & 0 & 0 & 11.2 & 7.07 & 124.32 & 13.07 & 7.33 & 150.41 & 0 & 0 & 0 & 11.87 & 12.53 & 15.33 & 0 \\
\hline & $\mathrm{V}$ & 0 & 9.87 & 4.5 & 14 & 7.87 & 1.78 & 0 & 0 & 11.87 & 7.73 & 144.06 & 13.6 & 8.27 & 176.58 & 0 & 0 & 0 & 12.27 & 13.13 & 0 & 0 \\
\hline & VII & 0.667 & 9.83 & 5.33 & 12.8 & 6.4 & 1.67 & 0 & 0 & 11.67 & 8.67 & 158.85 & 12.27 & 8.27 & 159.31 & 0 & 0 & 0 & 9.47 & 8.67 & 0 & 0 \\
\hline & IX & 0 & 0 & 0 & 12.47 & 0 & 0 & 0 & 0 & 0 & 0 & 0 & 0 & 0 & 0 & 0 & 0 & 0 & 0 & 0 & 0 & 16 \\
\hline \multirow{5}{*}{ 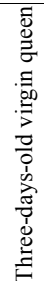 } & I & 0 & 0 & 0 & 10.87 & 6.13 & 0 & 0 & 0 & 11.07 & 7.73 & 134.35 & 10 & 6.67 & 104.72 & 0 & 0 & 0 & 9.6 & 10.8 & 12.13 & 0 \\
\hline & III & 0.667 & 8.8 & 4 & 10.8 & 6.27 & 2.2 & 6 & 4 & 10.93 & 7.6 & 130.42 & 10.67 & 7.73 & 129.49 & 0 & 0 & 0 & 8.27 & 10.53 & 10.8 & 0 \\
\hline & $\mathrm{V}$ & 0.667 & 9.07 & 4.2 & 11.33 & 6.67 & 2 & 0 & 0 & 11.47 & 7.2 & 129.66 & 12.53 & 8.4 & 165.25 & 0 & 0 & 0 & 8.93 & 11.33 & 0 & 0 \\
\hline & VII & 0.667 & 6.47 & 4 & 11.47 & 5.47 & 2.33 & 5.33 & 6 & 9.73 & 9.07 & 138.55 & 10.13 & 9.2 & 146.32 & 0 & 0 & 0 & 5.33 & 6.933 & 7.33 & 6.53 \\
\hline & IX & 0 & 0 & 0 & 14.67 & 0 & 0 & 0 & 0 & 0 & 0 & 0 & 0 & 0 & 0 & 0 & 0 & 0 & 13.6 & 0 & 0 & 13.8 \\
\hline
\end{tabular}


(Table 1B) contd....

\begin{tabular}{|c|c|c|c|c|c|c|c|c|c|c|c|c|c|c|c|c|c|c|c|c|c|c|}
\hline \multirow{4}{*}{ 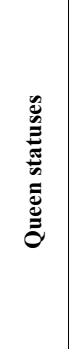 } & \multirow{4}{*}{ 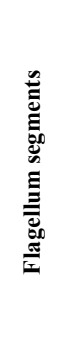 } & \multicolumn{21}{|c|}{ Measurements of sensilla organs } \\
\hline & & \multirow{3}{*}{ 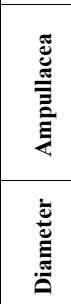 } & \multirow{3}{*}{ 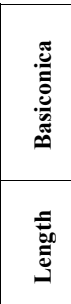 } & \multirow{3}{*}{ 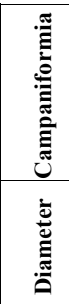 } & \multicolumn{2}{|c|}{ Chaetica } & \multicolumn{3}{|c|}{ Coeloconica } & \multicolumn{9}{|c|}{ Placodea } & \multicolumn{4}{|c|}{ Tricodea } \\
\hline & & & & & \multirow{2}{*}{ 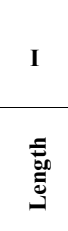 } & \multirow{2}{*}{ 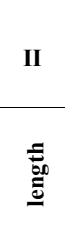 } & \multirow{2}{*}{ 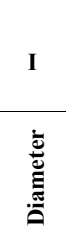 } & \multicolumn{2}{|c|}{ II } & \multicolumn{3}{|c|}{ I } & \multicolumn{3}{|c|}{ II } & \multicolumn{3}{|c|}{ III } & \multirow{2}{*}{ 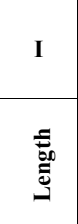 } & \multirow{2}{*}{ 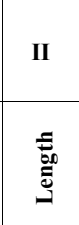 } & \multirow{2}{*}{ 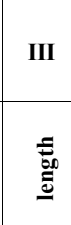 } & \multirow{2}{*}{ 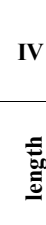 } \\
\hline & & & & & & & & 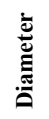 & 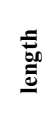 & 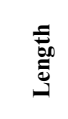 & $\frac{ \pm}{3}$ & 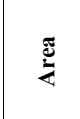 & 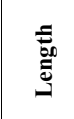 & 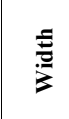 & 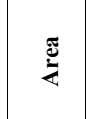 & $\begin{array}{l}\bar{E} \\
\bar{\Xi} \\
\vec{\Xi}\end{array}$ & $\frac{E}{E}$ & $\stackrel{\Xi}{z}$ & & & & \\
\hline \multirow{4}{*}{ 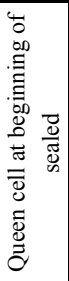 } & I & 0 & 0 & 0 & 12.67 & 8.4 & 0 & 0 & 0 & 11.07 & 7.07 & 122.88 & 10.8 & 7.6 & 128.87 & 0 & 0 & 0 & 12.4 & 11.6 & 14 & 0 \\
\hline & III & 0 & 0 & 0 & 12.53 & 7.6 & 0 & 0 & 0 & 12 & 7.33 & 138.09 & 10.67 & 4.53 & 75.89 & 0 & 0 & 0 & 10.67 & 11.33 & 0 & 0 \\
\hline & $\mathrm{V}$ & 0 & 6 & 0 & 0 & 6.13 & 0 & 0 & 0 & 11.47 & 7.6 & 136.86 & 11.07 & 7.87 & 136.78 & 0 & 0 & 0 & 8.87 & 9.6 & 0 & 0 \\
\hline & VII & 0 & 0 & 0 & 10.2 & 5.47 & 2 & 0 & 0 & 10.53 & 8.13 & 134.41 & 10.27 & 7.47 & 120.45 & 0 & 0 & 0 & 7.5 & 7.33 & 0 & 0 \\
\hline \multirow{4}{*}{ 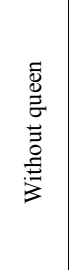 } & III & 0.667 & 6.63 & 0 & 0 & 5.6 & 0 & 0 & 0 & 8 & 6 & 75.36 & 9.73 & 7.73 & 118.08 & 0 & 0 & 0 & 6.45 & 8 & 9 & 0 \\
\hline & $\mathrm{V}$ & 0 & 7.07 & 0 & 0 & 6.67 & 0 & 0 & 0 & 8.67 & 6 & 81.67 & 8.93 & 7.2 & 100.95 & 0 & 0 & 0 & 0 & 0 & 7.47 & 0 \\
\hline & VII & 0 & 6.2 & 0 & 9.73 & 6 & 0 & 0 & 0 & 9.33 & 8 & 117.19 & 9.33 & 8.27 & 121.014 & 0 & 0 & 0 & 0 & 6.13 & 0 & 0 \\
\hline & IX & 0 & 0 & 0 & 13.33 & 0 & 0 & 0 & 0 & 0 & 0 & 0 & 0 & 0 & 0 & 0 & 0 & 0 & 0 & 0 & 0 & 14.8 \\
\hline \multirow{4}{*}{ 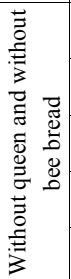 } & I & 0.667 & 10.33 & 4.67 & 11.78 & 8 & 0 & 0 & 0 & 10.4 & 6.4 & 104.49 & 9.6 & 6.93 & 104.45 & 0 & 0 & 0 & 13.6 & 12.93 & 13.73 & 0 \\
\hline & III & 0 & 0 & 0 & 15.07 & 0 & 1.33 & 0 & 0 & 10.4 & 7.33 & 119.68 & 10.67 & 7 & 117.26 & 0 & 0 & 0 & 14.13 & 14.93 & 0 & 0 \\
\hline & $\mathrm{V}$ & 0 & 0 & 0 & 14.4 & 0 & 0 & 0 & 0 & 11.87 & 7.47 & 139.21 & 12.4 & 6.13 & 119.34 & 0 & 0 & 0 & 14 & 14.53 & 0 & 0 \\
\hline & VII & 0 & 11.67 & 0 & 13.33 & 10 & 1.78 & 0 & 0 & 12.8 & 8 & 160.77 & 12.13 & 8 & 152.35 & 0 & 0 & 0 & 15 & 14.93 & 0 & 0 \\
\hline
\end{tabular}

c- Sensilla Campaniformia (Cf) didn't occur on the different flagellomeres in the queen cell and queenless statuses. They didn't reveal on the first and ninth flagellomeres in the one-year-old mated queen, supersedure queen, and threedays-old virgin queen statuses, but few numbers of these sensilla were distributed on the other different flagellomeres. These sensilla ranged from 4 to $5.33 \mu \mathrm{m}$ in diameter in all statuses.

d- The long Chaetica (Ch I) is relatively ascended on most of the different flagellomeres of the workers under all queen statuses, with varying in number distribution. The $(\mathrm{Ch}$ I) didn't appear on the fifth flagellomere of the workers under the queen cell status, and the third and fifth (without queen status). The lowest number of these sensilla exhibited in the queenless status. These sensilla ranged from 9.73 to $16.67 \mu \mathrm{m}$ in length in all statuses. While, the short Chaetica (Ch II) didn't exist on the ninth flagellomere of the workers under all queen statuses; and also the third and fifth in the without queen/without bee bread status. The highest total number of these sensilla appeared in the without queen status, but the lowest number appeared in the without queen/without bee bread status. These sensilla ranged from 5.47 to $10 \mu \mathrm{m}$ in length in all statuses.

e- Sensilla Coelloconica with very slightly protrusive peg (CO I), didn't uprise on the different flagellomeres under without queen status. Also they didn't uprise on the first and ninth flagellomeres of the workers under all queen statuses and the fifth in the without queen/without bee bread status, but in the queen cell status, they indicated only on the seventh flagellomere. These sensilla ranged from 1.33 to $2.4 \mu \mathrm{m}$ in diameter in all statuses. Whereas, the Coelloconica with longest peg (CO II) exhibited in extremely very few numbers on the first flagellomere only in the supersedure queen status; and on the third and seventh flagellomeres in the three-day-old virgin queen status, but they didn't appear on the different flagellomeres of the workers under the other queen statuses. These sensilla ranged from 2.67 to $6 \mu \mathrm{m}$ in diameter and its pig ranged from 2 to $6 \mu \mathrm{m}$ in length in all statuses.

f- Sensilla Placodea type one (PL I) were absent on the ninth flagellomere of the workers under all queen statuses, while their distribution varying on the other different flagellomeres. These sensilla ranged from 75.36 to $160.77 \mu \mathrm{m}^{2}$ in area, where the smallest one appeared in the without queen status and the largest one appeared in the without queen/without bee bread status. Sensilla Placodea type two (PL II) didn't ascend on the ninth flagellomere of the workers under all queen statuses. The highest total number of these sensilla appeared in the queenless status. The (PL II) ranged from 89.02 to $189.39 \mu \mathrm{m}^{2}$ in area, where the smallest one appeared in the supersedure queen status and the largest one appeared in the one-year-old mated queen status. 


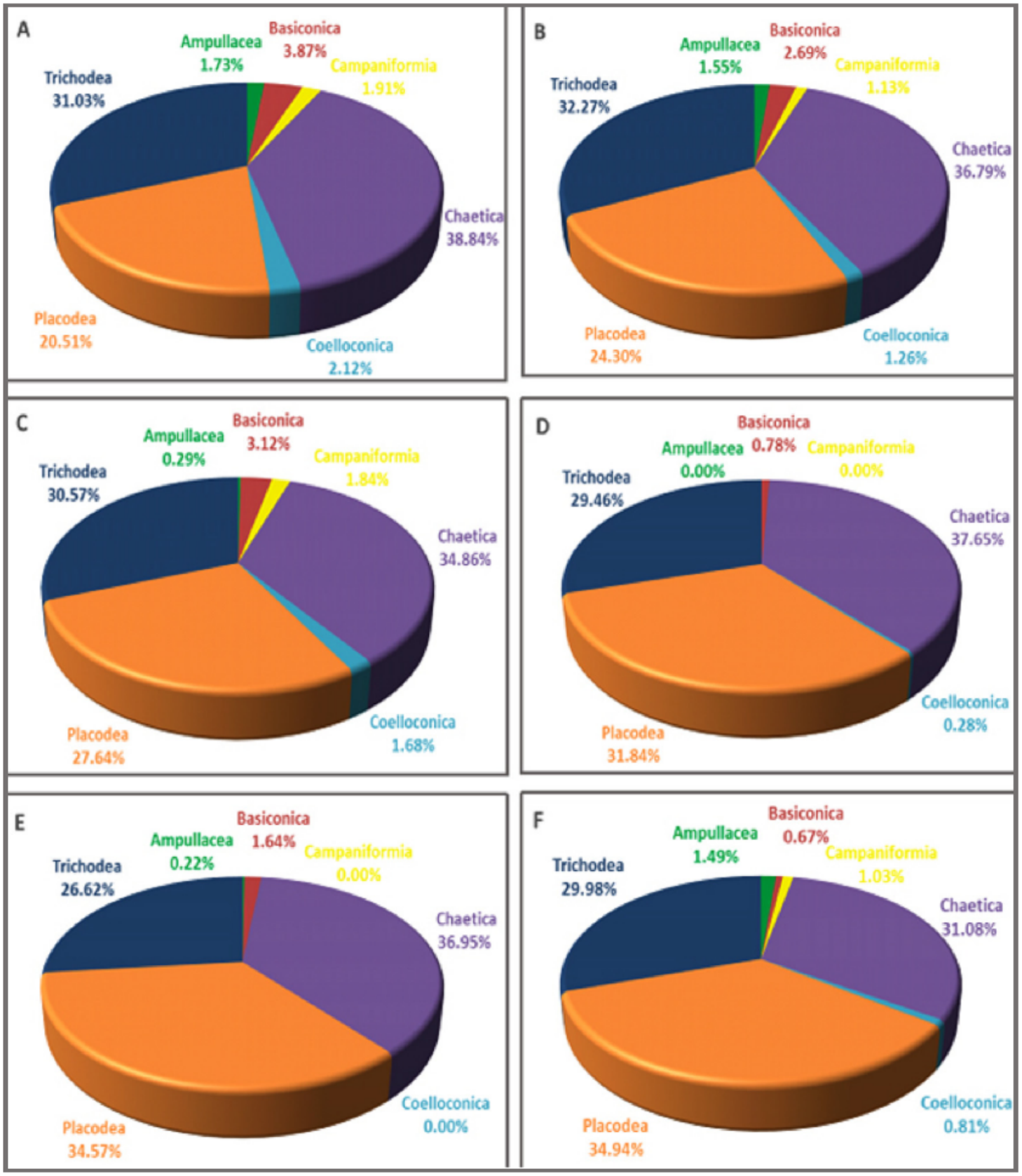

Fig. (3). Percentages of antennal sensilla per unit area $(122 \mu \mathrm{m} \times 82 \mu \mathrm{m})$ on the dorsal sides of different flagellomeres of Apis mellifera workers at three-day-old under different queen statuses. A: one-year-old mated queen, B: three-days-old virgin queen, C: supersedure queen, D: queen cell, E: without queen, and F: without queen/ without bee bread.

On the other hand, sensilla Placodea type three (PL III) isn't produced on the different flagellomeres of the workers under all queen statuses.

g- Sensilla Trichodea type one $(\operatorname{Tr} I)$ were subsisted on the most of the different flagellomeres of the workers under all queen statuses with varying in the number distribution. These sensilla ranged from 5.33 to $18.27 \mu \mathrm{m}$ in length in all statuses, where the shortest one appeared in the three-daysold virgin status and the longest appeared in the without queen/without bee bread status.

However, Sensilla Trichodea type two (Tr II) indicated on the most of the different flagellomeres of the workers under all queen statuses with varying in the number distribution. They were more abundant on the first flagellomere (the tip of the antenna) in most queen statuses, except in the case of the one-year-old mated queen status, where the third flagellomere has the highest number of these sensilla. These sensilla ranged from 6.13 to $14.93 \mu \mathrm{m}$ in length in all statuses, where the shortest one found in the queenless status and the longest in the without queen/without bee bread status. While, sensilla Trichodea type three ( $T r$ III) distributed in few numbers and ranged from 7.33 to $15.33 \mu \mathrm{m}$ in length in all statuses, where the shortest one appeared in the three-daysold virgin queen status and the longest appeared in the supersedure queen status. On the contrary, sensilla Trichodea type 

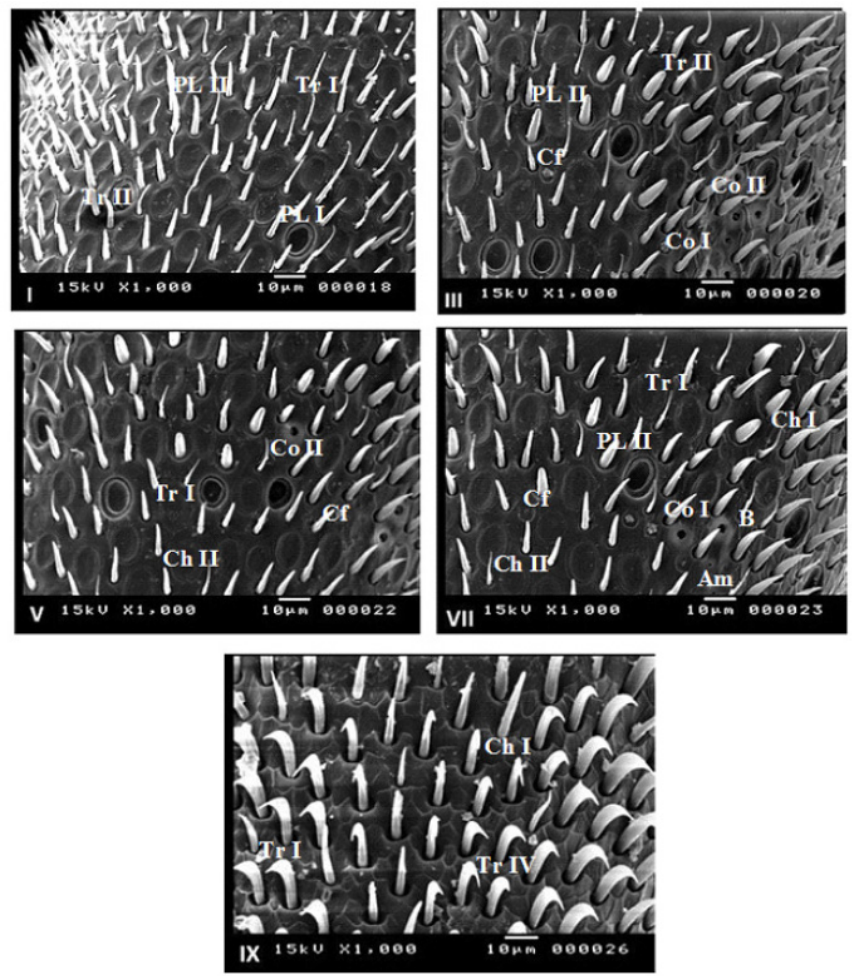

Micrograph (3A). The different sensilla per unit area $(122 \mu \mathrm{m} x$ $82 \mu \mathrm{m})$ on the dorsal side of different flagellomeres (I, III, V, VII, IX) of Apis mellifera workers at three-day-old under one-year-old mated and egg-laying queen.
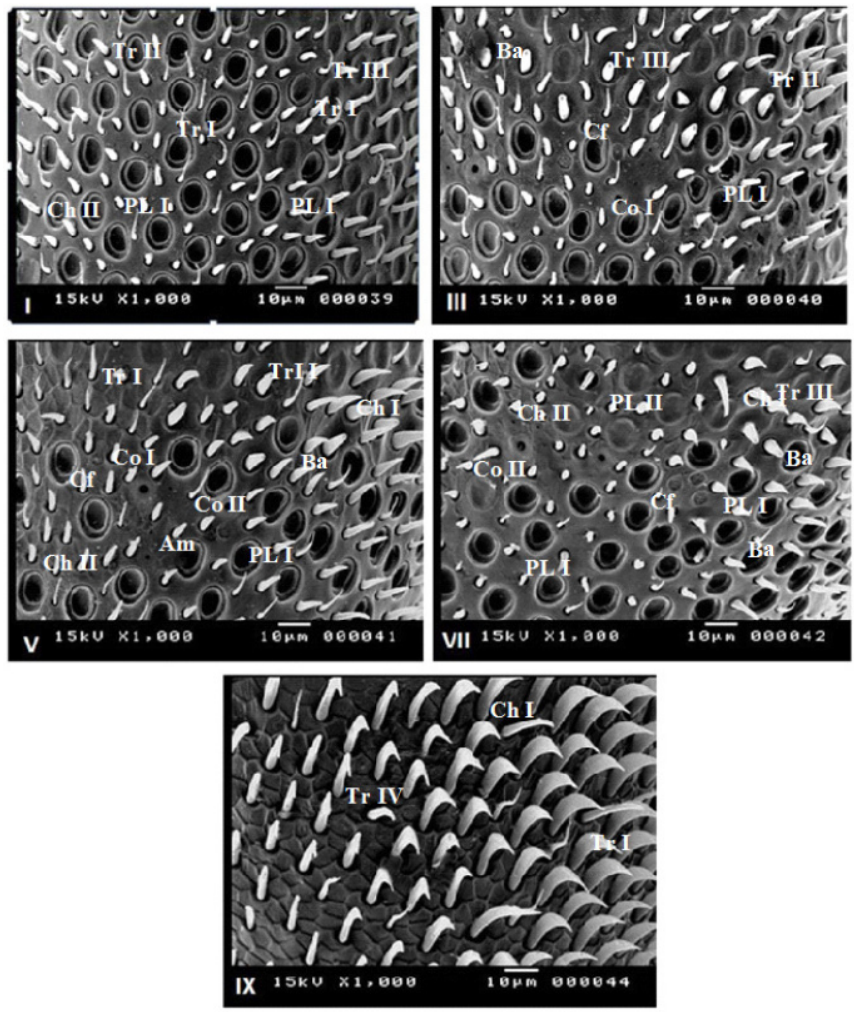

Micrograph (3C). The different sensilla per unit area $(122 \mu \mathrm{m} x$ $82 \mu \mathrm{m}$ ) on the dorsal side of different flagellomeres (I, III, V, VII, IX) of Apis mellifera workers at three-day-old under three-day-old virgin queen.
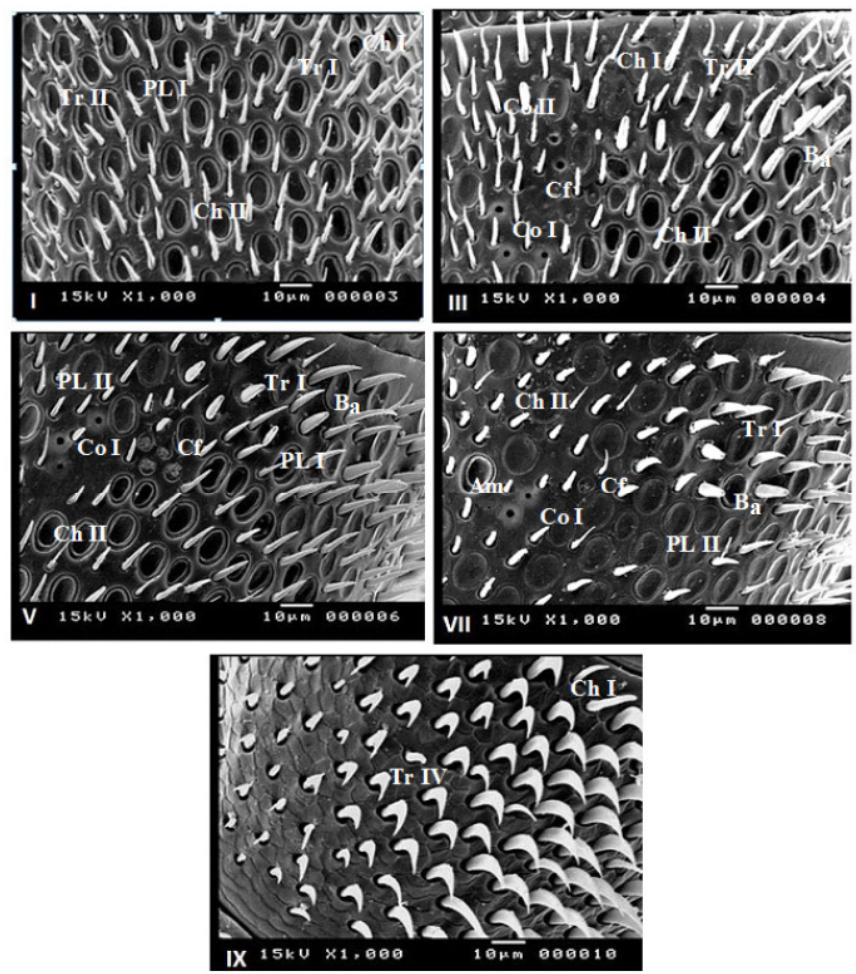

Micrograph (3B). The different sensilla per unit area $(122 \mu \mathrm{m} x$ $82 \mu \mathrm{m}$ ) on the dorsal side of different flagellomeres (I, III, V, VII, IX) of Apis mellifera workers at three-day-old under supersedure queen.
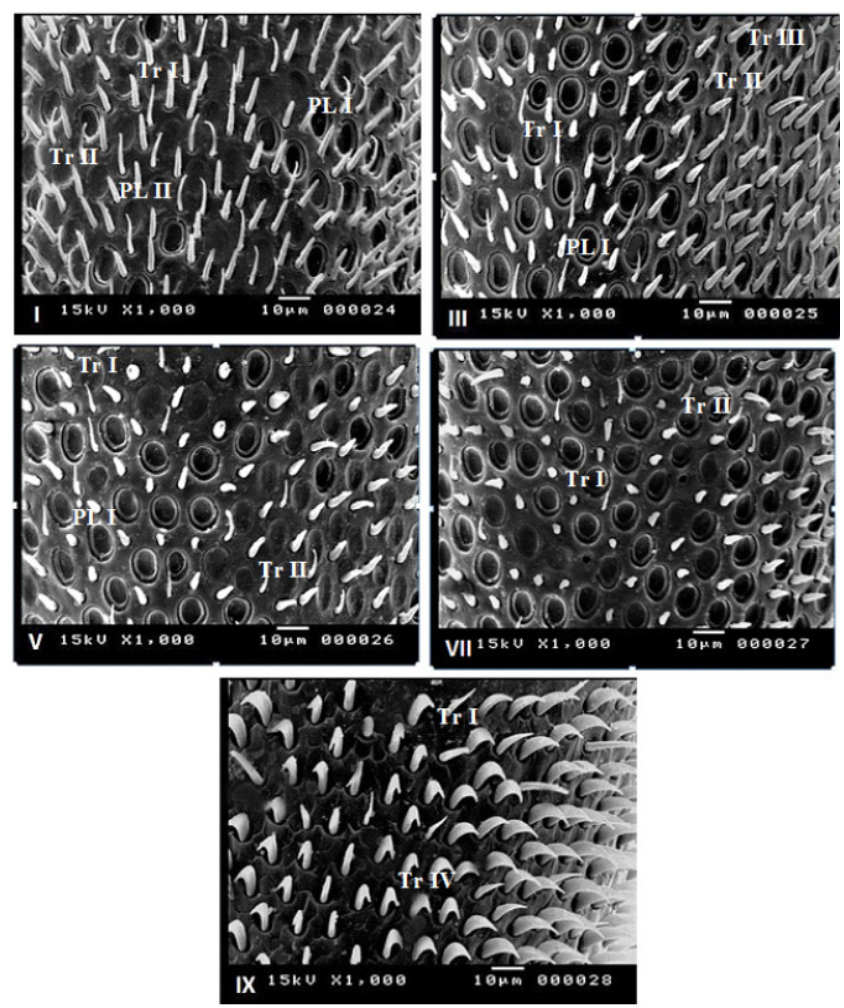

Micrograph (3D). The different sensilla per unit area $(122 \mu \mathrm{m} x$ $82 \mu \mathrm{m}$ ) on the dorsal side of different flagellomeres (I, III, V, VII, IX) of Apis mellifera workers at three-day-old under queen cell at beginning of sealed. 

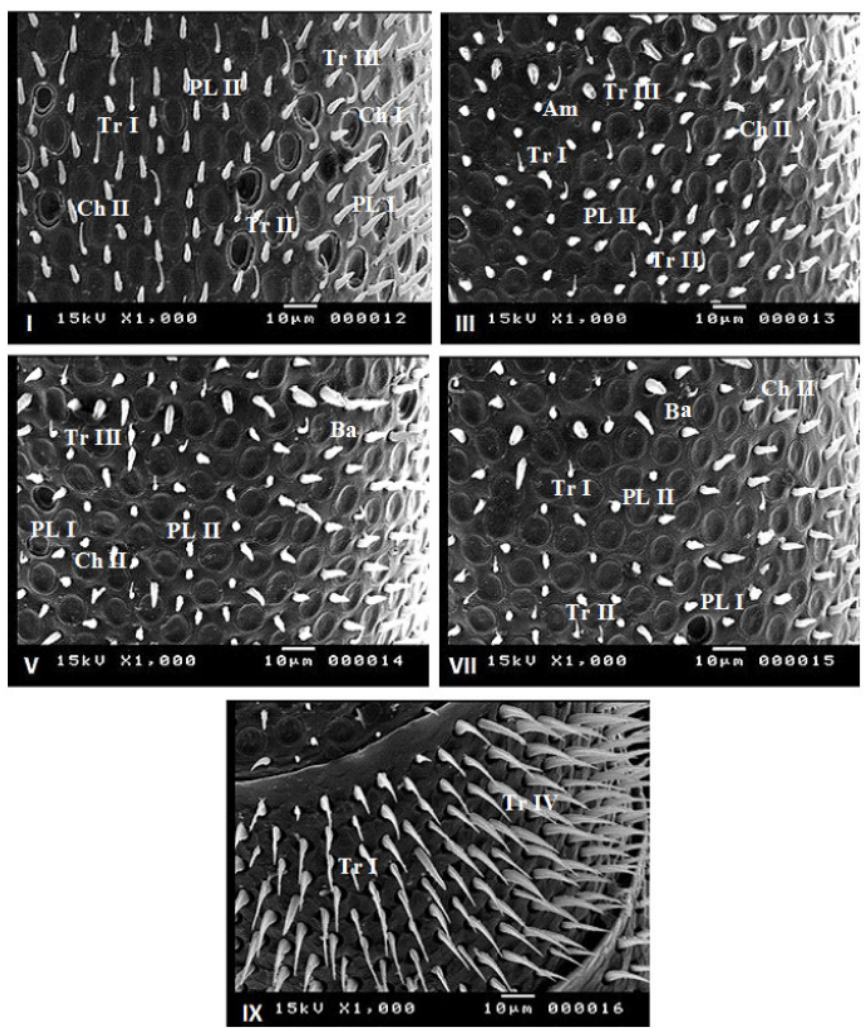

Micrograph (3E). The different sensilla per unit area $(122 \mu \mathrm{m} x$ $82 \mu \mathrm{m}$ ) on the dorsal side of different flagellomeres (I, III, V, VII, IX) of Apis mellifera workers at three-day-old without queen.

four (Tr IV) appeared only on the ninth flagellomere of the workers under nearly all queen statuses, but in the threedays-old virgin queen status they appeared on the seventh and ninth flagellomeres. In all statuses, the total numbers of (Tr IV) were more than the other sensilla Trichodea types. These sensilla ranged from 6.53 to $19.2 \mu \mathrm{m}$ in length in all statuses.

\section{DISCUSSION}

\subsection{Morphological Characters of the Workers}

\section{A. Determination of Morphological \& Ultrastructural Studies of the Workers Antennae}

The antennae are the main sensory organs of the bee for smelling, tasting, and hearing, as well as detecting changes in temperature, vibration, wind, and humidity. There are a variety of the sensory organs (sensilla) on the different flagellomeres of the honeybee workers and this as previously indicated by [33], that the behavior of the bees are influenced by external stimuli detected by sensory organs.

The cumulative evidence by [34], the age dependent and size dependent polyethism of the honeybee workers play a great role in the morphology and maturation, so that differences in sensory sensivity depend on the bee's maturation or age.

In our study, workers flagellum at three day-old was examined by SEM. Large numbers of the different sensilla occurred on the dorsal side of the different flagellomeres of the workers. Workers at (3-18) days-old were examined accord-
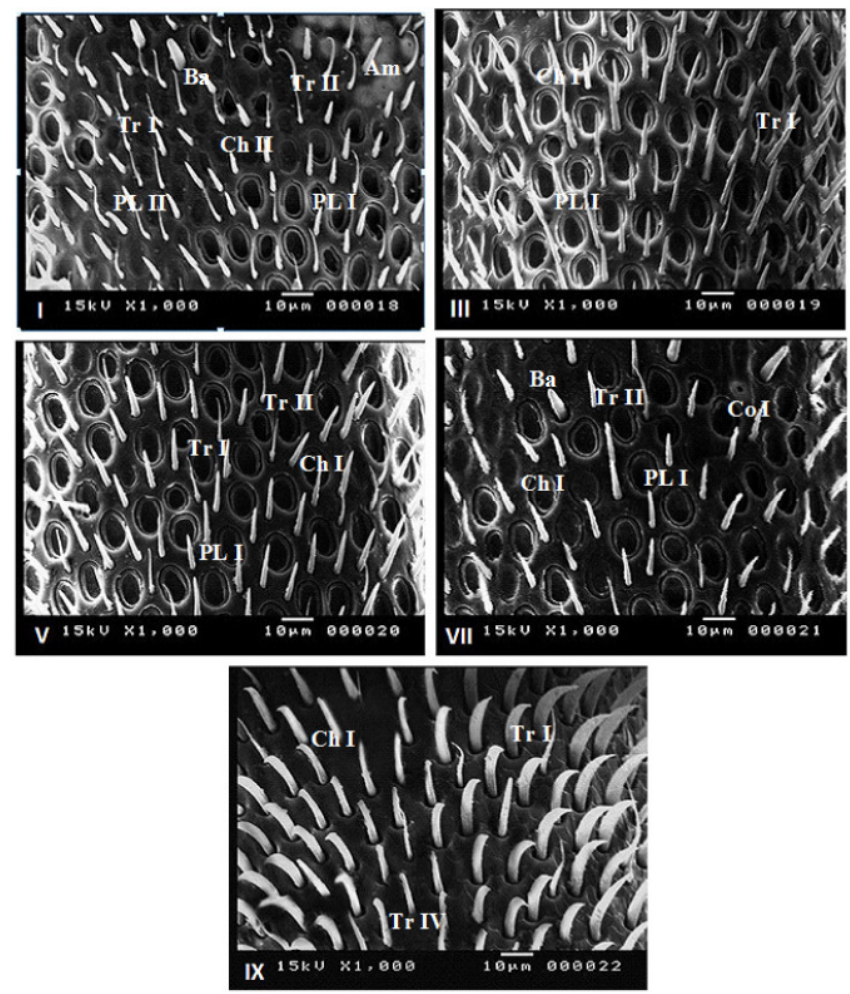

Micrograph (3F). The different sensilla per unit area $(122 \mu \mathrm{m} x$ $82 \mu \mathrm{m}$ ) on the dorsal side of different flagellomeres (I, III, V, VII, IX) of Apis mellifera workers at three-day-old without queen and without bee bread.

ing to $[35,20-23]$; they found that, bees showed a poor learning performance to odours during the first 2 days of age. Later workers achieved a higher level of acquisition, $i$. e. on the $3^{\text {rd }}-4^{\text {th }}$ day; it became equal to that of older bees.

Honeybee queens produce vital pheromones that regulate colony organization and worker morphology, behavior and physiology $[7,8]$. These pheromones depend on the status of the queen. [36], Proved that, workers in the presence of a queen have improved performance in olfactory learning and memory assays compared to bees in queenless colonies. This is agreeable with that proved by [37] they said, a mated queen releases pheromones that exert a profound influence on the most important life activities of the colony. The queen loss in a honeybee colony influences workers' behavior and the sensitivity of their antennal receptors to queen extract odour, or affects the maturation of the antennal lobes of the worker bee brain, etc.

\section{A.1. The Different Sensilla Organs of Apis mellifera Work-} $\underline{\text { ers }}$

The electrone microscopic study of the honeybee workers Apis mellifera at three-day-old, headed by different queen statuses (one-year-old mated queen, supersedure queen, three-days-old virgin queen, queen cell, without queen, and without queen/without bee bread) in our study, revealed the presence of seven types and eleven subtypes of sensilla on the dorsal side different flagellomeres (I, III, V, VII, IX) of the workers; those are: sensilla Ampullacea $\{\mathrm{Am}\}$, sensilla Basiconica $\{\mathrm{Ba}\}$, sensilla Campaniformia $\{\mathrm{Cf}\}$, sensilla Chaetica I, II $\{\mathrm{Ch}\}$, sensilla Coelloconica I, II $\{\mathrm{Co}\}$, sensilla 
Placodea I, II, III $\{$ PL $\}$, and sensilla Trichodea I, II, III, IV $\{\operatorname{Tr}\}$ (Micrograph 1). These results have a remarkable similiration to that reported by [27], whereas they classified insect sense organs as following:

Sensilla Trichodea, sensilla Chaetica, sensilla Basiconica, sensilla Coeloconica, sensilla Ampullacea, sensilla Campaniformia, and sensilla Placodea. Also, these different sensilla have previously been indicated by [38] from scanning electron microscopic (SEM) studies of worker honeybees, Apis mellifera. Many other Hymenopterans had many sensilla types that located on the flagellum, sensilla Ampullacae (a receptor for carbon dioxide); Basiconica (olfactory sensilla); Campaniformia (mechanoreceptors); sensilla Chaetica; sensilla Coeloconica; sensilla Placodea, (odour receptors); Trichodea type A (olfactory sensilla); Trichodea type B and C (mechanoreceptors), and Trichodea type D (gustatory receptors) [9-12, \& 39, 40]. The results of the present study are almost agreed with those reported by $[13,14]$, where the micro-morphology of the antennae of the honeybee Apis florea $\mathrm{F}$., the types, number and distributions of the various types of sensills were examined. He found that, the location of different types of sensilla on antenna of Apis florea is similar to those of Apis mellifera, also the distribution of various types of sensilla along the antenna is similar to those found in other Apis species. Six types of the sensilla organs were recorded; sensilla Placodea, Basiconica, Trichodea, Ampullacea, Coeloconica and Campaniformia. Moreover, he reported that, sensilla Trichodea found most frequently on all antennal segments. Sensilla Placodea and sensilla Basiconica were present on eight distal segments. Sensilla Ampullacea and sensilla Coeloconica were present on five distal segments, while, sensilla Campaniformia were confined to seven distal segments. The highest density of sensilla was found on the distal segment.

[41] recorded the following sensilla, Ampullaceaous, Basiconica, Campaniforme, Placodae and Trichodae (type A, B, C\&D) are the most abundant at the distal ends of the flagellum of Apis dorsata. Also, this study relatively agree with the data obtained by [42] who found six types of the sensilla organs; Trichodea type (A\&B), Placodea, Basiconica, Coeloconica and Campaniformia in the flagellomeres No. $2,4,6,8 \& 10$ of the worker honeybee antennae as Apis mellifera and Apis florea. Similar findings were according to [43] preliminary observations; he distinguished the following sensillum types:

Sensilla Placodea, sensilla Trichodea, sensilla Basiconica, sensilla Coeloconica (referred also to as Ampullacea), sensilla Campaniformia or coelocapitular and sensilla Chaetica. He also articulated the sensillenmuster, the numbers and measurements of the different sensilla, differe according to the difference in the workers' age and the queen statuses. The results agreed with those recorded by [30], who indicated that, these types of sensilla are: sensilla Trichoidea, sensilla Chaetica, sensilla Basiconica, sensilla Coeloconica, sensilla Ampullacea, sensilla Campaniformia and sensilla Placodea, that have been traditionally classified on the basis of the morphology of their cuticular parts, as well as the location on the insect.

While, these results varied somewhat with that reported by $[44,45]$ who identified ten types of the sensilla organs in antennal dwarf honeybee workers of Apis florea F., these variations indicated Basiconic peg, Campaniform, Chaetica (type1, 2, 3 \&4), Coeloconica, Falcate, Placodea and Trichodea.

\section{A.1.1. Sensilla Ampullacea (Am)}

According to this present study, sensilla Ampullacea (Am) didn't appear on segment IX of the workers under all queen statuses, that their function is olfactory, according to [43], there are no olfactory receptors on the first two proximal $(9,10)$ segments of the honeybee flagellum or olfaction is lost upon amputation of the 8 distal segments. The lowest percentages appeared in the workers at 3-days-old in the supersedure queen and queenless statuses.

This study revealed that, there is no significant difference in the numbers of sensilla Ampullacea, and the measurement of sensilla (Am) is constant, where their diameter is 0.667 $\mu \mathrm{m}$ of the workers under all the queen statuses, this may be a result of the experimental conditions, where the bee cages were incubated at a constant temperature $32^{\circ} \mathrm{C} \pm 1$ and a constant humidity $60 \% \mathrm{RH}$. conditions, but we can indicate the relatively boorish difference of (Am) numbers according to its location on the distal ends of the eight distal flagellomeres; according to their function; as the bee's maturation or age; the queen status and the effect of the bee bread diet.

During the first days of the bee adult life, high levels of spontaneous responses might be related to a higher sensitivity to the odours of queen pheromones or the external environment. Poor learning performance of young might be a result of absence of the queen pheromones or the low quantities of these pheromones according the queen status, also for example, the workers under supersedure queen status, where the percentage of (Am) is low, but as we indicated because the experimental bee cages incubated at constant temperature and constant humidity conditions the difference between the percentages isn't segnificant.

Our findings suggests that, sensilla (Am) are olfactory or smell chemoreceptor involved in perception of temperature, carbon dioxide and humidity, this is agreeable with [46]. [47], was the first who recorded impulses from a hygroreceptor (a moist receptor) on an antenna of the honeybee Apis mellifera. According to the previous results recorded by [48] in Apis mellifera, the surface around the pore of the sensilla Ampullacea was patterned. These have been shown to respond to carbon dioxide. Also, $[49,50]$ identified the sensilla Ampullacea as $\mathrm{CO}_{2}$ receptors.

\section{A.1.2. Sensilla Basiconica (Ba).}

Sensilla (Ba) didn't appear on segment IX of the workers under all queen statuses, their distribution concentrated on the segments III, V and VII, but it appeared in low concentrations on the first segment that, it is contact chemoreceptor sensitive to gustative stimuli and it sometimes worked as tactile mechanoreceptors. The highest percentages of $(\mathrm{Ba})$ appeared on the workers at 3-days-old in the one-year-old mated queen status, while, the lowest percentages appeared in the queen cell at beginning of sealed and without queen/without bee bread statuses.

Our results revealed that, there was no significant difference in the percentages of numbers of $(\mathrm{Ba})$ between the dif- 
ferent queen statuses of workers. This can be explained according to their location on the eight distal flagellomeres, their function, the workers' maturation or age, the queen status and the effect of the bee bread diet. So that, Sensilla (Ba) are chemoreceptors for taste (Gustatory or contact chemoreceptor) and for smell (Olfactory or smell chemoreceptor) and also mechanoreceptors.

$[27,28]$ revealed that, sensilla Basiconica are chemical receptors. Also as indicated by [51] that, certain gustatory sensilla take the form of pegs (basiconic sensilla). [4] proved that, workers may change between different tasks depending on their physiological maturation or age.

Similar findings were reported by [30] that sensilla Basiconica can be solely mechano-, contact chemo-, and olfactory rreceptors. Contrary to them our results vary with that of (Ba) can be also thermo- and hygrosensitive in function.

\section{A.1.3. Sensilla Campaniformia (Cf).}

Sensilla Campaniformia (Cf) didn't appear on segment IX in all the ages of the workers under all queen statuses. The highest percentages of (Cf) appeared on the workers at 3 -days-old in the one-year-old mated queen and the supersedure queen statuses. This study assumed that, there is no significant difference in the percentages of (Cf) between the different queen statuses of workers. This can be explained according to their location on the eight distal flagellomeres, their function, the workers' maturation or age, the queen status and the effect of the bee bread diet. So that, sensilla (Cf) are considered in general as mechanoreceptors and / or hygro - and thermoreceptors sensitive to temperature, $\mathrm{CO}_{2}$, humidity or a combination of these factors. Hence, there is an association between sensilla (Am) and (Cf).

As we indicated before, the experimental bee cages incubated at constant conditions; so that, the difference between the percentages of $(\mathrm{Cf})$ numbers aren't significant and the measurements are relatively constant and we can indicate the boorish difference in the measurements to their mechanical function beside the olfactoty and the hygro - thermal function.

The absence of (Cf) during the young ages of the workers might be due to, the absence of the queen pheromones as in the absence of queens' statuses; or due to the affecting of these pheromones as in the queen cells status.

From the previous results in Apis mellifera, workers and males have similar numbers of sensilla Campaniformia and sensilla Ampullacea. Since sensilla Ampullacea are considered to be hygroreceptor organs [46].

The electrophysiological findings of [47] for Apis mellifera, that sensilla Campaniformia is sensitive to temperature, carbon dioxide and humidity or a combination of these factors. Also, these results are agreeable with those obtained by $[10,51-53]$. The data obtained by [54] agreed with our obtained results for that, the sensillum coelocapitulum (campaniform sensilla), a hygro- and thermoreceptive sensillum of the honeybee, Apis mellifera. The results indicated by [55] found correlation between numbers of the Campaniformea and Ampullacea + Coeloconica sensilla organs and the defense behavior in Africanized honeybees, that means (Cf) are mechano and olfactory in function.
However, these results differ somewhat from previous results by [10] where they found that, the presence of campaniform sense organ on at least 9 of the 10 segments of the honeybees' flagellum suggests that its function is not olfactory, because it is known that olfaction is lost upon amputation of the 8 distal segments, but they found the campaniform sensilla, in general, are considered to be mechanoreceptors, and the possibility that the campaniform sensilla are sensitive to temperature, $\mathrm{CO}_{2}$, humidity, or a combination of these factors is discussed.

\section{A.1.4. Sensilla Chaetica: (Ch) I, II}

In this study the two types of sensilla Chaetica (Ch I and Ch II) appeared on the segment IX of the workers under certain queen statuses so that, their function isn't olfactory. The distribution of both (Ch) I and (Ch) II are concentrated on the segments I, III, V and VI, that means sensilla Chaetica are tactile mecanoreceptors and also contact chemoreceptor sensitive to gustative stimuli. These conclusions have previously been indicated by [56-59] that the terminal flagellar antennomere of honeybee workers is covered with large taste hairs. [60] found that, the terminal flagellar antennomere is covered with numerous small hairs, which are assumed to be unmodal tactile hairs. [61] proved the gustatory sensilla are mostly located on the distal segments of the antennae. The measurements of sensilla Chaetica differ significantly according to, the different queen statuses; the shortest one appeared at the workers without queens $9.73 \mu \mathrm{m}$, because of the absence of the queen pheromones. According to our present study, there is significant difference in the percentages of each (Ch I) and (CH II) between the different queen statuses of workers. This can be explained according to their morphology, location on the different flagellomeres, their function, the workers' age, the queen status and the effect of the bee bread diet.

The two types of sensilla Chaetica (Ch I) and (CH II) are contrasting in most statuses, so that they may be functionally completed to each other, sensilla (CH: I, II) are mechanoreceptors to tactile stimuli and contact chemoreceptor sensitive to gustative stimuli, moreover, they are considered as auditory organ, probably (Ch $\mathrm{I})$ are considered as taste hairs and also as auditory organs, while (CH II) are considered as tactile hair only. One could assume in this study, the effect of the bee bread diet could be indicated according to the gustatory function of sensilla Chaetica, where the highest percentage of the long Chaetica (Ch I) is present in the without queen/without bee bread status and this may be for searching the food (bee bread).

[62] showed that, the long Chaetica responded to sugar, fatty acids and alcohols and also to air $[57,58]$ identify sensilla Chaetica and sensilla Basiconica as gustatory sensilla one head and legs of honeybee. [63], found that, bristles (sensilla Chaetica) act as receptors for touch and air flow, they can also act as chemoreceptors. [59] showed that, antennal chaetic sensilla are very sensitive to sucrose stimulation. [30] defined the sensilla Chaetica- as hairs similar to the sensilla Trichodea, but have thicker cuticular and they can be mechano- or contact chemosensitive in function. [61] indicated that, taste is crucial for honeybees for choosing profitable food diet sources, resins, water sources, and for nestmate recognition. 


\section{A.1.5. Sensilla Coeloconica: (Co) I, II}

The two types of sensilla Coeloconica (Co I and Co II) didn't appear on the segment IX on the flagellum of the workers and under all queen statuses.

According to this study, the highest percentages of (Co I) appeared on the workers at 3-days-old in the one-year-old mated queen status; While, the lowest percentages appeared in the workers at the queen cell, and without queen/without bee bread statuses. The numbers and distributions of (Co II) were exhibited at extremely low percentages in the different statuses. The (Co II) is shown on the workers at only 3-daysold in the supersedure queen and three-days-virgin queen statuses.

Our results found that, there is no significant difference in the numbers of (Co I), and also in the measurement of their diameters and pegs. This may be a result of the experimental conditions, where the bee cages were incubated at constant temperature $32^{\circ} \mathrm{C} \pm 1$ and constant humidity $60 \%$ $\mathrm{RH}$. conditions, but we can indicate the relatively boorish difference of (Co I, II) numbers according to their location close to the distal ends of each the eight distal flagellomeres; according to their function; the bee's maturation or age; the queen status and the effect of the bee bread diet.

Sensilla Coeloconica are associated with sensilla (Am) and sensilla (Cf); where sensilla (Co I, II) are olfactory or smell chemoreceptor involved in perception of temperature, carbon dioxide and humidity.

These results are similar with [46], that both the sensilla Coeloconica and sensilla Ampullacea are considered to be hygroreceptor organs. In recordings from their vicinity, [47] found (sensilla Coeloconica and sensilla Ampullacea) are clustered together in small groups close to the distal ends of the eight distal flagellar segments. Also, [55] found correlation between numbers of the Campaniformea and Coeloconica sensilla organs and the defense behavior in Africanized honeybees.

\section{A.1.6. Sensilla Placodea: (PL) I, II, III}

All subtypes of sensilla Placodea (PL: I, II, III) didn't appear on the segment IX in the workers under all queen statuses. The sensilla Placodea upraised in very high numbers on the different flagellomeres (I, III, V and VII) and they are varying in the distribution between these flagellomeres. The highest percentages of (PL I) appeared on the workers at the supersedure queen, three-days-virgin queen, queen cell, and without queen/without bee bread statuses. While, the lowest percentages appeared in the workers without queen status. According to our present study, we noted that, the percentages of (PL II) higher than the percentages of (PL I) in the most statuses. There is significant difference in the percentages of each (PL I, II, III) between the different queen statuses, this can be explained according to their morphology, location on the 8-distal flagellomeres, their function, the workers' age, the queen status and the effect of the bee bread diet.

Our findings suggesting that, sensilla Placodea provide sense of smell (smell chemoreceptor), they are considered the main olfactory sensilla in honeybee and also they can measure the air pressure. The highest percentages of each
(PL I); (PL II); and (PL III) is detected in the without queen status, this may be due to the olfactory function of sensilla Placodea, searching for queen pheromones.

The workers at 3-days-old under the queenright statuses, where one-year-old mated queen status have relatively low percentage of (PL I) (3.02\%), and in the three-days-old virgin queen status the workers have relatively low percentage of (PL II) $(5.88 \%)$, but high percentages of sensilla placodea appeared at the prime ages in the other queenright statuses; this can be illustrated as the high levels of spontaneous responses during the first days of adult life that might be related to higher sensitivity to the odours, when the sensory environment (queen pheromones) could affect the setting up of the olfactory system. While, relatively low percentage of (PL I) appeared at 3-days-old in the without queen status $2.63 \%$.

$[66,67]$, recorded the same results on Apis mellifera, the sensilla Placodea have been shown to be odour receptors. According to, [51], the main olfactory sensilla of honeybee Apis mellifera are the sensilla Placodea. [22] found the olfactory system is still maturing during the first days of the adult bee life (the youngest bees). [37] proved a great improvement in olfactory learning of queenright workers during the first three days of adult life. The olfactory learning in queenless worker bees reached its maximum only on the $10^{\text {th }}$ day.

\section{A.1.7. Sensilla Trichodea: (Tr) I, II, III, IV}

The most subtypes of sensilla Trichodea (excluding $\operatorname{Tr}$ III), are existed on IX flagellomere in certain statuses so that, their function isn't olfactory, while (Tr III) have an olfactory function because they disappeared on IX flagellomere in all statuses.

According to the present results, we noted that, the (Tr I, II, III, IV) are found in all statuses, so that they may be functionally completed to each other. There is significant difference in the percentages of each sensilla Trichodea types between the different queen statuses. This can be explained according to their, location on the different flagellomeres, their function, the workers' age, the queen status and the effect of the bee bread diet.

Sensilla Trichodea are considered as mechanoreceptors for tactile, chemoreceptors for smell (olfactory organs) and chemoreceptor for taste (gustatory organs) and also they can be auditory organ.

The low percentage of ( $\operatorname{Tr} I)$ at the prime of ages in most statuses may be due to the bee's maturation or age, where the workers are found close to the queens. Specitically, sensivity may depend on the location, size, number or structure of sensory organs; where (Tr IV) are concentrated mainly on the IX flagellomere (near to the pedicel) so that, their function can be auditory beside the mechano- and chemo- functions, because the auditory organ (Johnson's organs) found in the second segment of the antenna (the pedicel). The measurements of (Tr I, II, III, IV) associated with each other, depending on the association in their functions, whereas, they differ significantly according to the different queen statuses. The longest type is (Tr IV) and this may be according to their location on the IX flagellomere, and their specialization in the auditory function; where the longest ( $\mathrm{Tr}$ IV) is found at workers under the queen cell status. 
The effect of the bee bread diet could be indicated in the without queen/without bee bread status where the lowest percentages of ( $\mathrm{Tr}$ III) and (Tr IV) is produced in this status. The sensations of taste based on contact with chemoreceptors as the (Tr III) and (Tr IV). The olfactory function of sensilla Trichodea was hypothesized by various honeybee workers by $[2,10,68]$. Also, [38] proved that, the morphology of the trichodum sensillum indicates an olfactory function. However, [47] reported that sensilla Trichodea type A did not respond to any chemical stimuli, but sensilla Trichodea type B responded to mechanical stimuli in Apis mellifera.

Chemical signals (queen pheromones) play a great role in the colony communication of honeybees, within and outside the hive $[67,69]$.

The results obtained by $[56,51]$ on Trichodea D in Apis, are in a highly contrast to our results, where they proved that, the sensilla trichodea D only identified as contact chemosensitive sensillum on the honeybee antenna, and these results are similar with our obtained results with a very poor variations where this type of arrangement of these sensilla was comparable to that reported in our study. It was reported that the sensilla Trichodea and Placodea are present in the highest amount in Apis [63, 70]

The principal significance of our findings is that, during the first days of adult life improvements in olfactory and other learning performance in the honeybee correlate in time with the increased sensitivity of workers' antennal receptors to queen pheromone because during the first days; more and more bees join the retinue of the queen i. e. their behavior changes [15-17].

According to [71], the Johnson's organs, which, in the honeybee, perceive movement of antennal flagellum, response to movements of air and considered as flight speed indicator and this appeared in our results as there is no significant difference in the (Tr IV) in most status. [72] indicated, Sensilla Trichodea discribed as mechanosensory function with some also chemosensory and/or thermosensitive functions. However the results of [14] differ from our results; he concluded that, sensilla Trichodea (type A) is considered the most common structures on the antenna.

One could conclude that, the highest numbers of the different types of the sensilla appeared in sensilla Chaetica, Placodea and Trichodea, is due to their important functions for honeybee social organization. Some other previous results are fluctuated up and down above our obtained results according to the rearing condition or the used diet (The difference between their results and the present may be attributed to the variation in the tested food diet preparations), but they recorded the same conclusions. The function of different sensilla types located in honeybee workers, may be inferred by using the morphological criteria together with the effect of the queen statuses; and under the results obtained by previous authors.

\section{CONFLICT OF INTEREST}

The authors confirm that this article content has no conflict of interest.

\section{ACKNOWLEDGEMENTS}

Declared none.

\section{REFERENCES}

[1] Winston ML. The Biology of the Honeybee. Cambridge and London: Harvard University Press 1987; 66: pp. 181-98.

[2] Frisch KV. The Dance Language and Orientation of Bees. The Belknap press of Harward Univ. Press, Cambridge, Mass 1967; p. 566.

[3] Michener CD. The Social Behavior of the Bees. The Belknap Press of Harvard University Press, Cambridge, Mass 1974; p. 404.

[4] Johnson BR. Reallocation of labor in honeybee colonies during heat stress: the relative roles of task switching and the activation of reserve labor. Behav Ecol Sociobiol 2002; 51:188-96.

[5] Lindauer M. Ein beitrag zur frage der arbeitsteilung im bienenstaat. Z Vergl Physiol 1952; 34: 299-345.

[6] Oster GF, Wilson EO. Caste and Ecology in the Social Insects. Princeton: Princeton Univ Press 1978; p. 342.

[7] Slessor KN, Winston ML, Le-Conte Y. Pheromone communication in the honeybee (Apis mellifera L.). J Chem Ecol 2005; 31: 273145.

[8] Le-Conte Y, Hefetz A. Primer pheromones in social hymenoptera. Annu Rev Entomol 2008; 53: 523-42.

[9] Slifer EH. The structure of arthropod chemoreceptors. Annu Rev Entomol 1970; 15: 121- 42.

[10] Dietz A, Humphreys WJ. Scanning electron microscopic studies of antennal receptors of the worker honeybee, including sensilla Campaniformia. Ann Entomol Soc Am 1971; 64(4): 919-25.

[11] Norton WN, Vinson SB. A comparative ultrastructural and behavioural study of the antennal sensory sensilla of the parasitoid Cardiochiles nigriceps (Hymenoptera: Bracoidae). J Insect Morphol 1974; 142: 329-50.

[12] Agren L. Flagellar sensilla of some Colletidae (Hymenoptera, Apidae). Int J Insect Morphol Embryol 1977; 6: 137-46.

[13] Gupta M. Scanning electron microscopic studies of antennal sensilla of adult workers of Apis florea F. (Hymenopter: Apidae). Apidologie 1992; 23: 47-56.

[14] Wcislo WT. Sensilla numbers and antennal morphology of parasitic and non-parasitic bees (Hymenoptera: Apoidea)". Int J Insect Morphol Embryol 1995.

[15] Skirkevičius A, Skirkevičienè Z. Insect Chemorecept Vilnius Mokslas 1979; 4: 23-43.

[16] Vaitkevičienè G, Skirkevičius A. Orientatsiya nasekomykh i kleshchei. Izdvo Tomskogo Univ 1984; pp. 70-3.

[17] Pham-Delègue MH, Trouiller J, Bakchine E, Roger B, Masson C. Age dependency of worker bee response to queen pheromone in a four-armed olfactometer. Insects Sociaux 1991; 38: 283-92.

[18] Collins AM. Genetics of the response of the honeybee to alarm chemical, isopentyl acetate. J Apic Res 1979; 18: 285-91.

[19] Masson C, Arnold G. Ontogeny, maturation and plasticity of the olfactory system in the worker bee. J Insect Physiol 1984; 30: 7-14.

[20] Lopatina NG, Chesnokova EG, Dolotovskaia LZ, Woyke J. Influence of mutations which determine accumulations in the body of 3hydroxy kynurenine, on the behaviour and functions of the nervous system in honeybees. Ontogenesis 1985; 16(6): 616-9.

[21] Ray S, Ferneyhough B. The effects of age on olfactory learning and memory in the honeybee Apis mellifera. Neuro Rep 1997; 8: 78993.

[22] Laloi D, Gallois M, Roger B, Pham-Delègue MH. Changes with age in the olfactory conditioning performance of worker honeybees (Apis mellifera L.). Apidologie 2001; 32: 231-42.

[23] Algirdas S. Olfactory learning in worker honeybees from queenright and queenless colonies (Apis mellifera carnica Poll M.). Biology 2009; 55(3): 125-32.

[24] Arnold G, Masson C. Evolution, en fonction de l'âge, de la structure externe des sensilles olfactives de l'antenne de l'ouvrière d'abeille Apis mellifera L. C. R. Acad Sci Paris III 1981; pp. 292- 681.

[25] Winnington AP, Napper RM, Mercer AR. Structural plasticity of identified glomeruli in the antennal lobes of the adult worker honeybee. J Comp Neurol 1996; 365: 479-90.

[26] Awad AA. Ultrastructural and morphological discrimination of Adult, Pupal and larval stages of Spodoptera exigua, (Lepidoptera: 
Noctuidae), Ph.D. Thesis. Assiut. Faculty of Science, Assiut University 1999 .

[27] Snodgrass RE. Principles of insect morphology. Chapter XVII. M.Sc. Thesis. London, UK: Graw-Hill Book Company 1935.

[28] Snodgrass RE. Anatomy of the Honeybee. Ithaca, NY, USA: Comstock Publishing Associates.Cornell University Press 1956.

[29] Agren L. Flagellar sensilla of some Colletidae (Hymenoptera, Apidae). Int J Insect Morphol Embryol 1977; 6: 137-46.

[30] Méndez-Vilas A. Díaz J, Eds. Microscopy: Science, Technology, Applications and Education 2010; 322: 1001-7.

[31] Maurizio A. Pollener nährung und Lebensvorgänge bei der Honigbiene (Pollen nutrition and vital processes in the honeybee) (Apis mellifera L.). Landwirtsch Jahrb Schweiz Bienen-Zeitung 1954; 68(6): 115-28.

[32] Waller RA, Duncan DP. A bays rule for symmetric multiple comparison problem. Am Stat Assoc J 1969; 64: 1485-503.

[33] Warnke U. Effects of electric changes on honeybees. Bee World 1976; 57: 50-6.

[34] Andre JR. Sensory allomony, foraging task specialization and resource exploitation in honeybee. Behav Ecol Sociobiol 2010; 64: $955-66$.

[35] Lopatina NG, Dolotovskaya LZ. Effect of tryptophane and its metabolites on conditioned activity of the honeybee. J Higher Nervous Activity Zhurnal Vysshei Nervnoi Deyatel'nosti 1984; 34(5): 911-9.

[36] Morgan SM, Butz-Huryn VM, Downes SR, Mercer AR. The effects of queenlessness on the maturation of the honeybee olfactory system. Behav Brain Res 1998; 91(1): 115-26.

[37] Fan Y. Effects of queen mandibular pheromone on nestmate recognition in worker honeybees, Apis mellifera. Anim Behav 2010; 79(3): 649-56

[38] Slifer EH, Sekhon SS. Fine structure of the sense organs on the antennal flagellum of the honeybee, Apis mellifera Linnaeus. J Insect Morphol 1961; 109: 351-81.

[39] Schneider D. Basic problems of olfactory research. Tanyola CNT, Eds. In: Theories of Odour Measurement. U.K: Necmi N. Tanyolac 1968; pp. 201-11.

[40] Chapman RF. The insect structure and function. Colorcraft Kent 1982; p. 770.

[41] Suwannapong G, Wongsiri S. Scanning electron microscopic study of antennal sensilla of the giant honeybee workers, Apis dorsata $\mathrm{F}$. Bee for New Asia, $7^{\text {th }}$ Asian Apic. Association Conference and Tenth Beenet 2004; p. 1793.

[42] Al-Ghamdi AA. Scanning electron microscopic studies on antennal sensilla organs of adult honeybee workers in genus Apis (Hymenoptera: Apidae). Bull Entomol Soc Egypt 2006; 83: 1-11.

[43] Giorgio-Vallortigara FE. Lateralization in insects: theoretical and experimental approaches. Advisor: Ph.D. Italy: University of TRENTO -Center for Mind. Brain Sciences 2008; 53: 81-92.

[44] Amornsak W, Cribb B, Gordh G. External morphology of antennal sensilla of Trichogramma australicum Girault (Hymenoptera: Trichogrammatidae). Int J Insect Morphol Embryol 1998; 27: 6782 .

[45] Amornsak W, Sonong A, Phoosuwan K. An inventory of antennal sensilla from worker dwarf honeybee, Apis florea F. (Hymenoptera: Apidae). Thailand. International Bee Research Association. Cardiff 2000; pp. 143-50.

[46] Kuwabara M, Takeda K. On the hygroreceptor of the honeybee Apis mellifera. Physiol Ecol 1956; 7: 1-6.

[47] Lacher V. Elektrophysiologische Untersuchungen an einzelnen Rezeptoren für Geruch, Kohlendioxid, Luftfeuchtigkeit und Temperatur auf den Antennen der Arbeitsbiene und der Drohne (Apis mellifera). Z. Vergl Physiol 1964; 48: 587-623.

[48] Argen L. Comparison between air drying and critical point drying for SEM studies of the antennae of Apis mellifera (Hymenoptera: Apidae). Zoon 1975; 3: 213-38.
[49] Sötz U. Die Ultrastruktur der Sensilla Coeloconica und der Sensilla Ampullacea auf den Antennen von Apis mellifera. Diploma thesis, Lehrstuhl Biologie I, Naurwiss Fakultät III. Germany: University of Regensburg 1989.

[50] Kleineidam C, Tautz J. Perception of carbon dioxide and other "air-condition" parameters in the leaf cutting ant Atta cephalotes. Naturwissenschaften 1996; 83: 566-8.

[51] Esslen J, Kaissling KE. Zahl und Verteilung antennaler Sensillen bei der Honigbiene (Apis mellifera L.). Zoomorphologie 1976; 83: 227- 51.

[52] Yokohari F, Taneda H. Moist and dry hygroreceptors for relative humidity of the cockroach, Periplaneta Americana. J Comp Physiol 1976; 106: 137-52.

[53] Yokohari F, Tominaga Y, Tateda H. Antennal hygroreceptors of the honeybee, Apis mellifera. Cell Tissue Res 1982; 226: 63-73.

[54] Yokohari F. The coelocapitular sensillum, an antennal hygro- and thermoreceptive sensillum of the honeybee, Apis mellifera. Cell tissue Res 1983; 233: 355-65.

[55] Stort AC, Rebustini ME. Differences in the number of some antennal sensilla of four honeybee (Apis mellifera) type and comparisons with the defensive behavior. J Apic Res 1998; 37: 3-10.

[56] Martin H, Lindauer M. Sinnesphysiologische Leistungen beim Wabenbau der Honigbiene. Z Vergl Physiol 1966; 53: 372-404.

[57] Whitehead AT, Larsen JR. Electrophysiological responses of galeal contact chemoreceptors of Apis mellifera to selected sugars and electrolytes. J Insect Physiol 1976a; 22: 1609-16.

[58] Whitehead AT, Larsen JR. Ultrastructure of the contact chemoreceptors of Apis mellifera (Hymenoptera: Apidae). Int J Insect Morphol Embryol 1976b; 5: 301-15.

[59] Haupt SS. Antennal sucrose perception in the honeybee Apis mellifera L.: behaviour and electrophysiology. J Comp Physiol A 2004; 190: 735-45.

[60] Eichmüller S, Schäfer S. Sensory neuron development revealed by taurine immunocytochemistry in the honeybee. J Comp Neurol 1995; 352: 297-307.

[61] De Brito Sanchez MG. Taste Perception in Honeybees. Chem Senses 2011; 36(8): 675-92.

[62] Ruth E. Electrophysiologie dear sensilla Chaetica auf den antennan von periplaneta Americana. J Comp Physiol 1976; pp.105-55.

[63] Altner H. Insect sensillum specificity and structure: an approach to a new typology. Olfaction Taste 1977; 6: 295-303.

[64] Lacher V, Schneider D. Elektrophysiologischer Nachweis der Riechfunktion von Porenplatten (Sensilla Placodea) auf den Antennen der Drohne und der Arbeitsbiene (Apis mellifera L.). Z Vergl Physiol 1963; 47: 274-8.

[65] Vareschi E. Duftunterscheidung bei der Honigbiene-EinzelzellAbleitungen und Verhaltensreaktionen. Z Vergl Physiol 1971; 75: 143-73.

[66] Kaissling KE, Renner M. Specialized chemoreceptors in the pore plates of Apis. Z Vergl Physiol 1968; 59: 357-61.

[67] Free JB. Pheromones of social bees, New York: London, UK. Chapman \& Hall 1987; 218: pp. 29-68.

[68] Schneider D. Basic problems of olfactory research. Tanyola CNT, Eds. In: Theories of Odour Measurement 1968; pp. 201-11.

[69] Frisch KV. Tanzsprache und Orientierung des Bienen, Springer, Berlin [translated into English as The Dance Language and Orientation of Bees], Cambridge, Harvard University Press 1965; p. 372.

[70] Antonio C, Nilson B. Antennal sensory structure of Scaptotrigona postica, (Hymenoptera, Apidae). J Kans Entomol Soc 1981; 54(4): 751-6.

[71] Ai H, Nishino H, Itoh T. Feeling the vibes of the waggle dance. Topographic organization of sensory afferents of Johnston's organ in the honeybee brain. J Comp Neurol 2007; 502(6): 1030-46.

[72] Zacharuk RY, Shields VD. Sensilla of immature insects. Annu Rev Entomol 1991; 36: 331-54

Received: June 09, 2014

Revised: July 09, 2014

Accepted: July 25, 2014

(C) Awad et al.; Licensee Bentham Open.

This is an open access article licensed under the terms of the Creative Commons Attribution Non-Commercial License (http://creativecommons.org/licenses/by-nc/3.0/) which permits unrestricted, non-commercial use, distribution and reproduction in any medium, provided the work is properly cited. 